\title{
Multiscale failure analysis of periodic masonry structures with traditional and fiber- reinforced mortar joints
}

\author{
Fabrizio GRECO $^{(\mathrm{a})}$, Lorenzo LEONETTI ${ }^{(\mathrm{b})}$, Raimondo LUCIANO ${ }^{(\mathrm{c})}$, Patrizia TROVALUSCI ${ }^{(\mathrm{d})}$
}

(a) Department of Civil Engineering, University of Calabria, Rende, Italy, email: f.greco@unical.it

(b) Department of Civil Engineering, University of Calabria, Rende, Italy, email: lorenzo.leonetti@unical.it

(c) Department of Civil and Mechanical Engineering, University of Cassino and Southern Lazio, Rende, Italy, email: luciano@unicas.it

(d) Department of Structural and Geotechnical Engineering, Sapienza University of Rome, Rome, Italy, email: patrizia.trovalusci@uniroma1.it

Keywords: Masonry; Cohesive Fracture; Multiscale Modelling; Fiber reinforced mortar.

\begin{abstract}
In this paper, a novel adaptive multiscale model is proposed for accurately predicting the nonlinear mechanical response of periodic brick masonry due to crack initiation and propagation under general inplane loading histories. Such a model relies on a two-level domain decomposition technique, used in conjunction with an adaptive strategy able to automatically zoom-in the zones incipiently affected by damage localization, aimed at reducing the typically high computational effort associated with fully mesoscopic models. The proposed switching criterion is based on the numerical determination of mesoscopically informed first failure surfaces taking into account higher-order deformation effects. In order to assess the validity of the proposed strategy, several multiscale numerical simulations are carried out on a shear wall test by varying the required input parameters. An innovative application of the proposed multiscale model is then presented for investigating the role of the fiber content in fiber-reinforced mortars (FRMs), recently introduced for masonry construction and rehabilitation, on the overall response of a deep beam test.
\end{abstract}

\section{Introduction}

In the last decades, the need of mitigating the impacts of natural hazards on cultural heritage artefacts has inspired a huge number of repair and strengthening techniques for traditional masonry structures, also relying on the adoption of fiber-reinforced composite materials [1-6]. Fabric-reinforced cementitious matrix (FRCM) composite systems have emerged as a more sustainable alternative to the most used fiber-reinforced polymer (FRP) materials for restoration and rehabilitation of existing masonry buildings, especially those of historical and architectural interest [7-9]. The use of a cementitious matrix instead of a polymeric one allows for a better chemical, mechanical and aesthetical compatibility with the masonry substrate and offers a potential fire protection. Nowadays, reinforcing fibers are available in a wide variety of materials and may be grouped into four classes: steel (low carbon or stainless), mineral (glass, basalt, etc.), synthetic organic (carbon, aramid, PBO, etc.) and natural organic, including both plant and animal fibers. Among them, natural fibers are gaining more and more attention by virtue of their low cost and low environmental impact, in terms of levels of embodied energy and $\mathrm{CO}_{2}$ production. However, in the presence of exposed brickworks, strengthening of masonry with externally bonded composite materials is often not desirable and/or allowed, so that less intrusive rehabilitation techniques should be used. Recalling that the mortar joints are at the same time the weakest and the easiest to replace component in masonry structures, the process of repointing is 
widely advocated to restore structural stability and functionality of masonry without any loss in the original appearance of the building.

Recently, short fiber-reinforced mortar have been proposed for repointing, in order to achieve shrinkage control and rheological benefits [10-12], especially in the case of low-strength mortars (as natural hydraulic lime, or NHL mortars). Moreover, the use of low-modulus fibers (such as glass, polypropylene or basalt fibers) is particularly suited for achieving also significant toughness enhancements, which in turn leads to a more ductile damage behavior of masonry under both static and dynamic (e.g. seismic) actions [13,14]. In fact, the most significant enhancement resulting from the inclusion of short fibers in mortar is the improvement in post-cracking behavior, which is typically evaluated by its fracture energy [15]; it follows that cracking control is one of the most desired application of fiber-reinforced mortars to traditional masonries. The magnitude of the toughening effect of fibers on the overall mechanical behavior of fiberreinforced mortars (referred to as FRMs) has been proved to be depending on a number of factors, which can be grouped in three categories: (i) characteristics of matrix (binder type, aggregate type and size, solid fraction composition, water content); (ii) characteristics of fibers (fiber type and size); and (iii) characteristics of composite mixture (fiber content, distribution, and orientation). Accordingly, by varying any of these factors, different overall mechanical properties of masonry are obtained, in particular those driving its post-peak nonlinear response. A few experimental works exist in the literature, which are devoted to the investigation of the role of the FRM's microstructural properties on the macroscopic behavior of masonry under both monotonic and cyclic loads [13,14], but a systematic study of the importance of each microscopic property is still missing. In fact, a campaign of experimental tests which is able to completely investigate the influence of all the microstructural details of fiber-reinforced mortars on the global behavior of masonry under general loading conditions is impracticable.

More versatile approaches adopt analytical developments and/or numerical simulations, which are able to predict the peculiar mechanical behavior of a given masonry structure under arbitrary loadings; the accuracy of such approaches mainly depends on the level of detail available for the geometric and mechanical properties of masonry and its underlying microstructure. Indeed, if observed at the so-called mesoscopic scale, i.e. the scale of individual units, brick masonry material appears to be heterogeneous, being composed of bricks assembled by mortar joints according to a wide variety of alternative patterns. Because of its intrinsically heterogeneous nature, masonry material shows a strongly nonlinear overall response under general loading, usually accompanied by an increasing anisotropy level associated with the occurrence of different physical phenomena which take place at finer scales of observation (i.e. microcrack nucleation and coalescence, contact and friction between crack faces, void growth, etc.).

In the technical literature, two different classes of models have been extensively employed for investigating the mechanical behavior of masonry structures with traditional mortar joints, i.e. mesoscopic and macroscopic models. On the one hand, mesoscopic models may be considered the most accurate, since they predict the overall response of masonry explicitly taking into account all the mesoscopic structural details, but inevitably lead to complex nonlinear problems requiring a huge computational cost to be solved. The related analyses, often referred to as direct numerical simulations (DNSs), have been extensively performed by using classical finite element techniques, although there exist alternative interesting approaches such as the discrete element method (DEM), which is naturally developed in a dynamic setting; according to this approach, which is based on non-smooth contact dynamics (NSCD) [16], masonry units are modeled as rigid or deformable bodies interacting to each other by means of different interaction laws (see, for instance, [17]). The main advantage of the discrete element method over the finite element method is the possibility of handling the complete detachment of distinct bodies in a finite displacement and rotation setting as well as recognizing new contacts automatically during the simulation. On the other hand, macroscopic models consider all the mesostructural details smeared out in a homogeneous continuum, whose mechanical behavior is driven by nonlinear anisotropic constitutive laws of phenomenological type, mainly based on damage and/or plasticity models [18-20]; such models are more practice-oriented, leading to a notable reduction in time and memory requirements, but usually have more constitutive parameters to be calibrated; as a consequence, they are most valuable when a compromise between efficiency and accuracy is mandatory. 
In between mesoscopic and macroscopic models are the so-called multiscale models, which combine the advantages of the above-mentioned models, i.e. the computational efficiency of purely macroscopic models and the numerical accuracy of purely mesoscopic ones. These models adopt a macroscopic constitutive relation for masonry material which is not of a phenomenological kind, but is mesoscopically informed, i.e. directly arises from the mechanical behavior of the underlying constituents (units and mortar joints) as well as their mutual interactions at the mesoscopic scale. Most of the existing multiscale models are based on the concept of scale transition, according to which the information is passed from lower to higher scales within the theoretical frameworks of asymptotic homogenization and/or micromechanics [21-23]; depending on the type of coupling between the mesoscopic and the macroscopic problems, two groups of methods can be distinguished, i.e. sequential and semi-concurrent multiscale methods. The first ones establish a "one-way" coupling between the two scales, and therefore they are suitable for determining the macroscopic behavior of masonry in terms of stiffness and strength (see, for instance, [24] in which critical surfaces for running bond adobe masonry with straw fiber-reinforced bricks are derived), but have a limited predictive capability for problems involving damage; the latter ones involve a "two-way" coupling between the mesoscopic and the macroscopic scales, i.e. the homogenized properties have to be computed "on the fly" during the microstructural evolution due to damage and other nonlinear phenomena. Most of semi-concurrent multiscale methods, which assume an "exact" representation of damage at the fine scale, falls into the wide class of computational homogenization methods, also called global-local approaches; in a finite element setting, such methods are referred to as $\mathrm{FE}^{2}$ methods $[25,26]$. Such methods differ from each other in the underlying modeling hypotheses, including the constitutive response of the equivalent homogenized material; concerning this point, most of the existing homogenization strategies adopt a classical (Cauchytype) macro-continuum, but there exists some interesting refined multiscale approaches assuming a Cosserat or higher-order continuum at the macroscale, in order to predict more accurately the overall behavior of masonry (see [27-33]). Computational homogenization approaches have already been adopted by the authors for properly handling the problem of the ill-posedness of the homogenized macroscopic problem of a fiberreinforced composite material in the presence of both geometric (see [34-36]) and material (see [23,37]) instability associated with cracking phenomena.

A subgroup of semi-concurrent multiscale methods, collectively referred to as continuous-discontinuous homogenization approaches, incorporate an internal length into the homogenized model in order to regularize the macroscopic mechanical response of masonry in the presence of strain localization [38,39]. Nevertheless, semi-concurrent multiscale models require a high computational effort; therefore, a number of alternative approaches have been proposed in the recent literature, based on a simplified representation of the nonlinearity sources, including those based on mesoscopically informed damage and/or plasticity models (see [40-42]) and those employing the transformation field analysis (TFA) (see [43]).

In the present work, a totally different multiscale strategy is proposed for the failure analysis of periodic masonry, which abandons the concept of scale transition in favor of the concept of scale embedding, according to which any heterogeneous structural model is decomposed into a set of fine- and coarse-scale sub-models to be solved simultaneously, in the spirit of the well-known domain decomposition methods (DDMs). Such a strategy, which has already successfully applied to fiber-reinforced composites and concretes (see [44-46] and [47-49], respectively) has been used for masonry structures in the same authors' previous work [50] for the first time, to the best authors' knowledge. Encouraged by the promising preliminary results obtained in [50], a refined ad-hoc concurrent multiscale model is here proposed, able to perform complete failure analyses of masonry structures under general in-plane loading histories in a very accurate manner. Such a model relies on a two-level domain decomposition method, able to couple a homogenized coarse-level description in regions characterized by a homogeneous macroscopic deformation, whose properties are obtained by classical first-order homogenization techniques, and a fine-level description in regions characterized by damage localization phenomena, for which the hypotheses of separation of scales and periodicity are no longer valid. The main novelty with respect to [50] is the introduction of a refined zooming-in criterion to be used within the adaptive model refinement strategy, which is responsible for the efficiency of the entire multiscale method, allowing to automatically switch the model from the coarse- to the fine-level description during the damage evolution process. Such a criterion relies on the detection of the first 
failure for in-plane loaded periodic masonries by means of the determination of mesoscopically informed first failure domains defined in the macrostrain space, eventually reduced according to heuristic arguments in the presence of non-negligible higher-order deformation effects.

In order to validate the proposed strategy, firstly several multiscale numerical simulations are carried out for a masonry panel with traditional mortar joints subjected to a shear test; in particular, a parametric analysis is performed by varying the numerical parameters required by the proposed multiscale strategy, aimed at tuning them so that an optimum balance between computational efficiency and numerical accuracy is found.

Then the proposed multiscale model is applied to the failure analysis of masonry structures with fiberreinforced mortar joints; a series of numerical experiments are carried out for investigating the overall toughening effect associated with different fiber fractions for a deep beam structural configuration. The main advantage of the adopted strategy consists in obtaining a macroscopic response directly as a function of the microstructural details of fiber-reinforced mortar joints.

\section{General multiscale framework for the masonry failure analysis}

At the mesoscopic level, brick masonry has been extensively modeled in the literature as a two-phase composite structure, made of artificial units (i.e. bricks) and mortar joints. Limiting the attention to the case of regular masonry, in which equal-sized bricks are connected by only horizontal and vertical joints, such a structure is equipped with a periodic mesostructure, being regarded as the union of translated homotheties of a repeating cell (RC). Since the out-of-plane mechanical behavior of masonry is not considered in this paper, a 2D model reduction is performed, based on the assumption of plane stress conditions [51,52]. Both units and mortar joints are susceptible to be damaged under the action of external in-plane loads; the resulting nonlinear mechanical behavior is taken into account by inserting cohesive interface elements not only at mortar joints (which is the most common simplified meso-modeling approach) but also along predefined plane or curved paths inside the units.

\subsection{Two-scale variational formulation}

A masonry structure is here defined as a 2D composite body made of units and mortar joints, occupying a bounded open subset of $\mathbb{R}^{2}$, denoted as $\Omega$, whose external boundary $\partial \Omega$ is assumed to be Lipschitzcontinuous; $\partial \Omega$ can be partitioned into two disjoint portions $\partial_{u} \Omega$ and $\partial_{t} \Omega$ upon which Dirichlet and Neumann boundary conditions are imposed, respectively, such that $\partial_{u} \Omega \neq \varnothing$ in order to avoid any rigid-body motion (see Fig. 1a). A generic unit, occupying the region $\Omega_{i}(i=1, \ldots, N$ with $N$ the number of units) is assumed to be made of linearly elastic material, except in a finite number of regions with vanishing thickness, whose location is a priori known, degenerating to interfaces, collectively denoted as $\Gamma_{b, i}$, which are subsequently equipped with a cohesive zone model accounting for crack initiation and propagation inside the bricks; the mortar joints are modeled as zero-thickness cohesive interfaces placed in between the units, collectively denoted as $\Gamma_{m}$, accounting for crack initiation and propagation at brick/brick and/or mortar/brick interfaces. In the small deformation setting, and assuming negligible body and inertial forces, the mechanical response of the considered masonry structure up to failure can be obtained as the solution of a nonlinear boundary value problem expressed by the following weak formulation: find $\boldsymbol{u} \in U$ such that: 

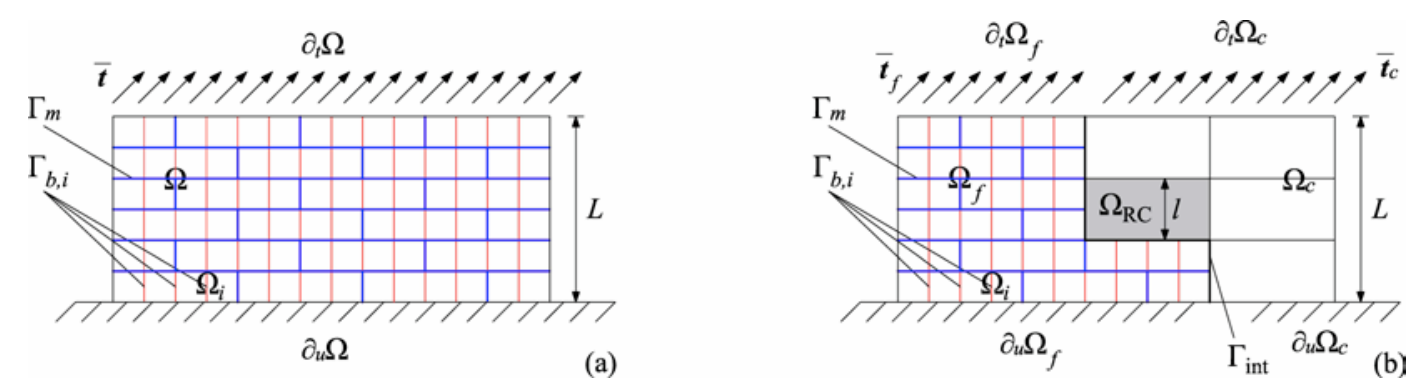

Fig. 1. Two-dimensional modeling approaches of a periodic masonry structure subjected to in-plane loading: (a) mesoscale model; (b) two-scale multi-domain model.

$$
\sum_{i=1}^{N}\left(\int_{\Omega_{i} \backslash \Gamma_{b, i}} \boldsymbol{\sigma} \cdot \boldsymbol{\varepsilon}(\boldsymbol{v}) d \Omega+\int_{\Gamma_{b, i}} \boldsymbol{t}(\llbracket \boldsymbol{u} \rrbracket, d) \cdot \llbracket \boldsymbol{v} \rrbracket d \Gamma\right)+\int_{\Gamma_{m}} \boldsymbol{t}(\llbracket \boldsymbol{u} \rrbracket, d) \cdot \llbracket \boldsymbol{v} \rrbracket d \Gamma=\int_{\partial_{t} \Omega} \overline{\boldsymbol{t}} \cdot \boldsymbol{v} d \Gamma, \forall \boldsymbol{v} \in V
$$

In Eq. (1), $\bar{t}$ is the traction applied on $\partial_{t} \Omega$, whereas $\boldsymbol{u} \in U$ and $\boldsymbol{v} \in V$ denotes respectively the (unknown) displacement and the (arbitrary) virtual displacement fields, which are defined in a standard manner; $\boldsymbol{\sigma}$ and $\boldsymbol{\varepsilon}$ are the stress and strain tensors, respectively, which are related to each other via a linearly elastic constitutive law describing the mechanical response of the brick material; moreover, the double brackets indicate the jump of the enclosed quantity, evaluated as the difference between of the values at the negative and positive sides of the cohesive interface; then, $\boldsymbol{t}$ is the cohesive traction vector acting on the positive side of the cohesive interface; finally, the scalar $d \in(0,1]$ denotes a state variable representing the current level of damage at the cohesive interface. The nonlinear mechanical problem represented by Eq. (1) is extremely complex to be solved in most of engineering applications, having a large ratio between the structural size and the brick size and, thus, involving a large number of units. In this paper, an adaptive multiscale strategy is proposed aimed at reducing the overall computational time, based on a two-level domain decomposition strategy, used in combination with a standard first-order homogenization technique.

The key idea of the proposed approach is to apply everywhere a classical first-order numerical homogenization scheme, after that the underlying mesostructure is replaced again at the critical hotspots of the homogenized numerical model; such hotspots are the regions subject to incipient cracking and/or influenced by boundary layer effects, for which both the assumptions of periodicity and separation of scales cease to be valid. It follows that the original single-scale single-domain problem is substituted by an equivalent two-scale multi-domain problem, composed of more manageable sub-problems to be solved in a fully coupled manner, which are defined on distinct subdomains and involving two separated spatial scales (a macroscopic and a mesoscopic scale). Without loss of generality, the original domain $\Omega$ is partitioned into two non-overlapping subdomains $\Omega_{c}$ and $\Omega_{f}$, as depicted in Fig. $1 \mathrm{~b} ; \Omega_{c}$ denotes the coarse-scale subdomain, representing the undamaged masonry, on which an equivalent homogenized material is assigned, whereas $\Omega_{f}$ is the fine-scale subdomain, representing the damaged masonry and called zone of interest, for which all the mesoscopic structural details are explicitly taken into account. This partition leads to the introduction of an additional internal boundary, referred to as meso/macro interface and denoted by $\Gamma_{\text {int, }}$ so that the variational problem (1) is restated as an interface variational problem based on the following Lagrange multiplier formulation: find $\left(\boldsymbol{u}_{f}, \boldsymbol{u}_{c}, \lambda\right) \in U_{f} \times U_{c} \times \Lambda$ such that:

$$
\begin{aligned}
& \sum_{i=1}^{N_{c}}\left(\int_{\Omega_{i} \backslash \Gamma_{b, i}} \boldsymbol{\sigma}_{f} \cdot \boldsymbol{\varepsilon}\left(\boldsymbol{v}_{f}\right) d \Omega+\int_{\Gamma_{b, i}} \boldsymbol{t}\left(\llbracket \boldsymbol{u}_{f} \rrbracket, d\right) \cdot \llbracket \boldsymbol{v}_{f} \rrbracket d \Gamma\right)+\int_{\Gamma_{m}} \boldsymbol{t}\left(\llbracket \boldsymbol{u}_{f} \rrbracket, d\right) \cdot \llbracket \boldsymbol{v}_{f} \rrbracket d \Gamma+\int_{\Gamma_{\mathrm{int}}} \lambda \cdot \boldsymbol{v}_{f} d \Gamma=\int_{\partial_{t} \Omega_{f}} \overline{\boldsymbol{t}}_{f} \cdot \boldsymbol{v}_{f} d \Gamma, \forall \boldsymbol{v}_{f} \in V_{f} \\
& \int_{\Omega_{c}}^{\boldsymbol{\sigma}_{c}} \cdot \boldsymbol{\varepsilon}\left(\boldsymbol{v}_{c}\right) d \Omega-\int_{\Gamma_{\mathrm{int}}} \boldsymbol{\lambda} \cdot \boldsymbol{v}_{c} d \Gamma=\int_{\partial_{t} \Omega_{c}} \overline{\boldsymbol{t}}_{c} \cdot \boldsymbol{v}_{c} d \Gamma, \forall \boldsymbol{v}_{c} \in V_{c} \\
& \int_{\Gamma_{\mathrm{int}}}^{\boldsymbol{\mu}} \boldsymbol{\mu} \cdot\left(\boldsymbol{u}_{f}-\boldsymbol{u}_{c}\right) d \Gamma=0, \forall \boldsymbol{\mu} \in \Lambda
\end{aligned}
$$


$\boldsymbol{u}_{f}$ and $\boldsymbol{u}_{c}$ being the coarse-scale and fine-scale displacement fields, respectively, and $\boldsymbol{v}_{f}$ and $\boldsymbol{v}_{c}$ the corresponding virtual fields. Moreover, $\overline{\boldsymbol{t}}_{f}$ and $\overline{\boldsymbol{t}}_{c}$ are the restrictions of $\overline{\boldsymbol{t}}$ to the portions $\partial_{t} \Omega_{f}$ and $\partial_{t} \Omega_{c}$ of $\partial_{t} \Omega$, respectively, whereas $\lambda$ represents the unknown Lagrange multiplier field and $\boldsymbol{\mu}$ its arbitrary variation. Finally, the macroscopic stress tensor is related to the macroscopic strain tensor via a suitably identified constitutive law, i.e. $\boldsymbol{\sigma}_{c}=\boldsymbol{C}^{\text {hom }} \boldsymbol{\varepsilon}\left(\boldsymbol{u}_{c}\right), \boldsymbol{C}^{\text {hom }}$ being the homogenized moduli tensor of undamaged masonry, to be determined as explained in Section 2.2.

\subsection{Mesoscopic modeling inside the zone of interest}

All the nonlinearities due to damage and fracture phenomena are assumed to occur exclusively inside the zone of interest, leading to the loss of validity of both periodicity and separation of scales in such a zone. As a consequence, an accurate mesoscopic modeling strategy is needed, able to include all the principal failure modes characterizing masonry, shown in Fig. 2: (a) mixed-mode cracking of mortar joints; (b) vertical tensile cracking of bricks; (c) diagonal tensile cracking of bricks; and (d) masonry crushing. The most common modeling approach is the so-called simplified meso-modeling, in which artificially expanded units are modeled by continuum elements whereas the behavior of both mortar joints and unit/mortar interfaces is lumped into discontinuous elements placed in between the units [53]; such an approach is unable to properly take into account failure modes (b) and (c). Enhanced modeling approaches exist, which consider potential cracks not only at mortar joints, but also inside the bricks along predefined lines running vertically in the middle of each unit (see, for instance, [54]). In the present work a refined mesoscopic modeling approach is proposed, able to take into account all the failure modes depicted in Fig. 2, which is based on the insertion of a number of crack planes with different location and/or orientation, in order to assure a more accurate match with an a priori unknown crack path. In particular, each unit is partitioned into 16 cross-triangle elements of equal dimensions arranged in a quadrilateral pattern, as shown in Fig. 3; the resulting internal boundaries are regarded as cohesive thin layers, i.e. as weak planes along which crack initiation and propagation may occur at pure mode I.

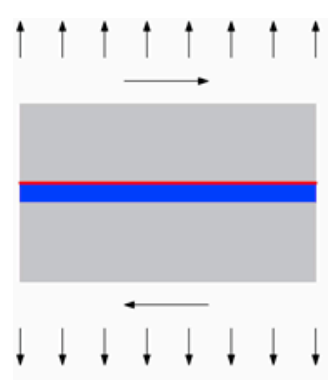

(a)

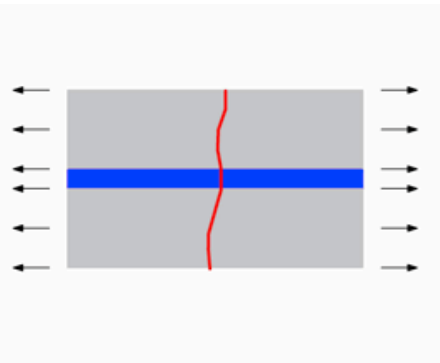

(b)

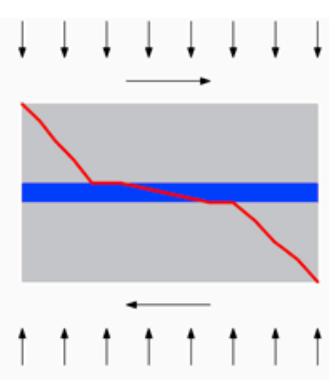

(c)

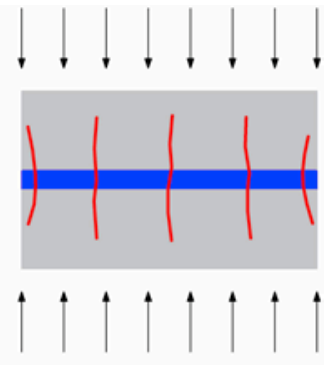

(d)

Fig. 2. Principal masonry failure modes: (a) mixed-mode cracking of mortar joints; (b) vertical tensile cracking of bricks; (c) diagonal tensile cracking of bricks; (d) masonry crushing.

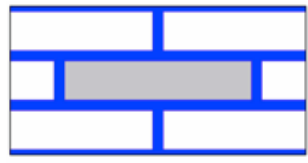

Mortar joints

Potential cracks in the bricks 
Fig. 3. Proposed mesoscopic modeling strategy, according to which expanded units are modeled as continuum elements, whereas both mortar joints and potential cracks in the units, arranged in a cross-triangle quadrilateral pattern, are modeled as zero-thickness cohesive interface elements.

All the considered damageable boundaries are equipped with the following 2D intrinsic cohesive law (see [55] for a comparative review of intrinsic and extrinsic cohesive zone models), based on the assumption of isotropic damage, and involving a scalar damage variable $d$ indicating the damage level of the interface:

$$
\boldsymbol{t}_{l}=(1-d) \boldsymbol{K}_{l} \llbracket \boldsymbol{u}_{l} \rrbracket,
$$

where $\boldsymbol{t}_{l}$ is the cohesive traction, $\boldsymbol{K}_{l}$ is the initial stiffness matrix of the (virgin) interface and $\llbracket \boldsymbol{u}_{l} \rrbracket$ is the displacement jump across the cohesive interface, all expressed with respect to the local frame of reference $(s, n)$; in the absence of cross coupling between normal and tangential directions, $\boldsymbol{K}_{l}$ turns to be diagonal, i.e. $\boldsymbol{K}_{l}=\operatorname{diag}\left(K_{s}, K_{n}\right)$, whose components are obtained differently for each interface type (brick interface or mortar joint interface). For the brick interfaces, $K_{s}$ and $K_{n}$ play the role of penalty stiffness parameters and must assume values sufficiently large to ensure displacement continuity before crack initiation but not too high to avoid convergence difficulties; conversely, for the mortar joint interfaces, $K_{s}$ and $K_{n}$ have a precise physical meaning, being derived from the properties of both masonry constituents and the joint thickness $h_{m}$, according to [56]:

$$
K_{s}=\frac{G_{b} G_{m}}{h_{m}\left(G_{b}-G_{m}\right)} ; \quad K_{n}=\frac{E_{b} E_{m}}{h_{m}\left(E_{b}-E_{m}\right)},
$$

where $E$ and $G$ denote the Young's and the shear moduli, respectively, and the subscripts $b$ and $m$ refer to the brick and mortar phases, respectively. The local undamaged stiffness matrix $\boldsymbol{K}_{l}$ can be transformed back into the global coordinate system via the transformation $\boldsymbol{K}=\boldsymbol{T}^{T} \boldsymbol{K}_{l} \boldsymbol{T}, \boldsymbol{T}$ being the orthogonal transformation matrix from the local to the global reference frame. In the present paper, an exponential softening response is adopted for both brick and mortar interfaces, associated with the following expression for the damage variable $d$ :

$$
d=\left\{\begin{array}{ll}
0 & \text { if } \delta_{\max }<\delta_{0} \\
1-\frac{\delta_{0}}{\delta_{\max }} \exp \left[-\frac{\delta_{\max }-\delta_{0}}{\frac{G_{c}}{\sigma_{t}}-\frac{\delta_{0}}{2}}\right] \quad \text { if } \delta_{\max } \geq \delta_{0}
\end{array},\right.
$$

$G_{c}$ and $\sigma_{t}$ denoting the mode-I fracture energy and the uniaxial tensile strength of the constituent (brick or mortar joint), respectively. Moreover, $\delta_{0}=\sigma_{t} / K_{n}$ is the critical displacement jump upon which the damage initiates, whereas $\delta_{\max }$ denotes the largest value ever attained by the equivalent displacement $\delta_{\text {eq }}$, which in turn is defined differently for brick or mortar interfaces. The nonlinear behavior of brick interfaces is supposed to be defined by a pure mode-I cohesive law, so that $\delta_{\text {eq }}$ coincides with the normal displacement jump $\llbracket u_{n} \rrbracket=\llbracket \boldsymbol{u} \rrbracket \cdot \boldsymbol{n}$; conversely, the mortar joint is equipped with a mixed-mode cohesive law derived from the degenerated Drucker-Prager criterion (including a compressive cap accounting for the crushing mechanism of masonry) proposed in [57], for which $\delta_{\text {eq }}$ depends also on the tangential displacement jump $\llbracket u_{s} \rrbracket=\llbracket \boldsymbol{u} \rrbracket \cdot s$. 


\subsection{Macroscopic modeling outside the zone of interest}

Outside the zone of interest, where the assumptions of periodicity and separation of scales are still valid, a standard numerical homogenization methodology is adopted to derive the overall (macroscopic) behavior of the undamaged masonry in terms of homogenized moduli tensor $\boldsymbol{C}^{\text {hom }}$. To this end, a linear mesoscopic variational problem defined over a suitably identified repeating cell (RC) is solved for the third-order tensor $\chi$ collecting the (unknown) characteristic functions $\chi^{p q}$ (with $p, q \in\{1,2\}$ ) corresponding to different macrostrain paths, assuming perfectly elastic interfaces and periodic boundary conditions.

The choice of such a repeating cell depends on the specific microstructure of masonry, but is not unique; referring exclusively to the running-bond pattern, any element shown in Fig. 4 may be used as a repeating cell for homogenization purposes; three families of RCs can be identified, which are arranged in a doubly periodic array according to rectangular $\left(C_{1}\right.$ to $\left.C_{8}\right)$, rhombic $\left(C_{9}\right.$ to $\left.C_{16}\right)$ and oblique $\left(C_{17}\right.$ to $\left.C_{24}\right)$ lattices, respectively. Among them, repeating cells $C_{9}$ to $C_{24}$ are associated with a complicated way to express periodic boundary conditions, if a classical rectangular coordinate system is used. As a consequence, in this work only rectangular-shaped cells have been considered, and specifically the type $C_{7}$ (or its mirror image, i.e. $C_{8}$ ) has been adopted, since in this case all the cohesive boundaries are internal boundaries, leading to some ease of implementation within the employed computational environment.

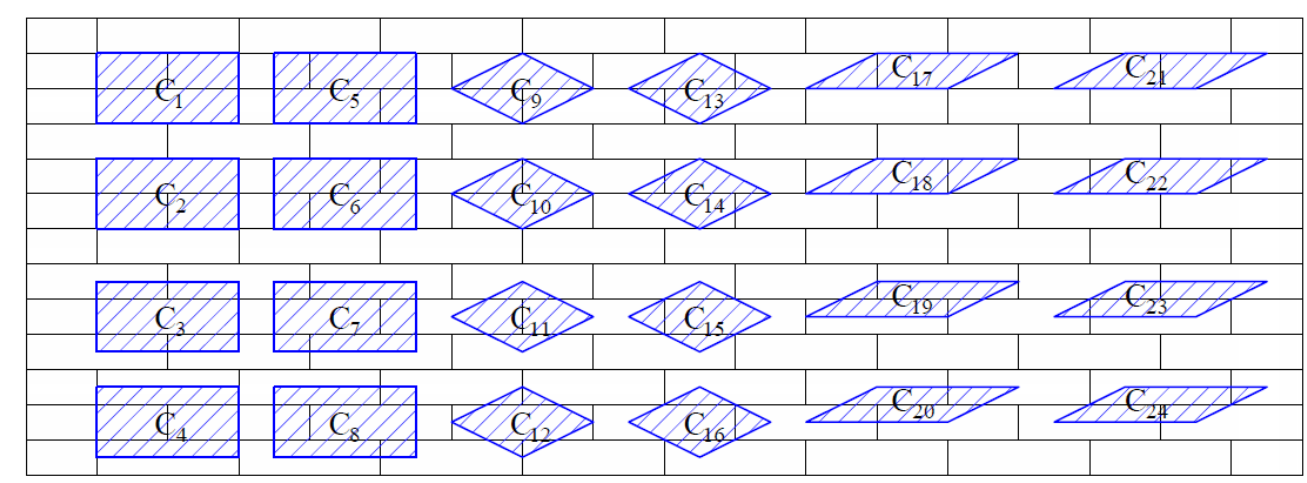

Fig. 4. Alternative repeating cells (RCs) for periodic masonry structures with running-bond brick pattern.

In a 2D setting under plane stress conditions, three separated (linear) mesoscopic problems are solved for the chosen repeating cell, subjected to two uniaxial and a shear paths, under periodic boundary conditions, as shown in Fig. 5.

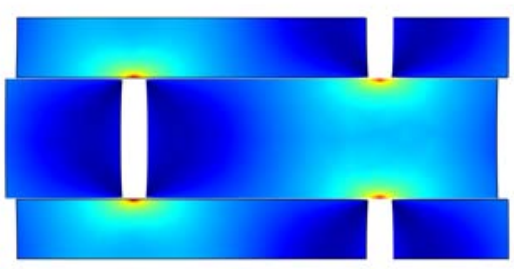

(a)

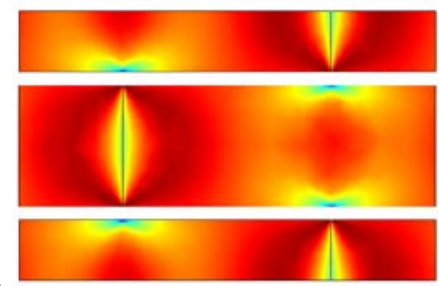

(b)

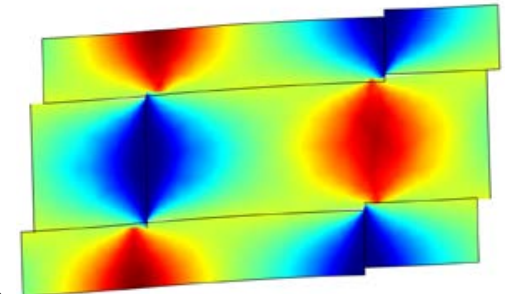

(c)

Fig. 5. Deformed configurations of the repeating cell for the undamaged masonry, subjected to three pure macrostrain modes, two uniaxial (a,b) and a shear (c) one, under plane stress and periodic boundary conditions. 
The resulting overall moduli tensor $\boldsymbol{C}^{\text {hom }}$ is expected to be orthotropic, with null moduli $C_{k k 12}^{\text {hom }}$; thus, only four overall moduli have to be identified, i.e. $C_{1111}^{\text {hom }}, C_{1122}^{\text {hom }}=C_{2211}^{\text {hom }}, C_{2222}^{\text {hom }}$ and $C_{1212}^{\text {hom }}$. These moduli will be assigned to the coarse-level subdomain outside the zone of interest, which is assumed to be not subjected to damage or fracture phenomena.

\subsection{Meso-to-macro connection within a FE setting}

An intrinsic feature of the multilevel domain decomposition strategy depicted in Section 2.1, if applied in a standard finite element setting, is the presence of a non-conforming mesh at the double-sided interface $\Gamma_{\text {int }}$. Since linear shape functions have been chosen for both coarse- and fine-scale displacement fields, each coarse interface element, whose length coincides with one of RC dimensions, shares only two nodes with the corresponding fine interface element, as shown in Fig. 6. These nodes are referred to as "corner nodes" whereas the nonmatching nodes are usually called "hanging nodes"; in the spirit of the dual-primal FETI method [58], the displacement continuity at corner nodes is enforced pointwise by using a primal method, such that a Lagrange multiplier method is employed only to enforce the compatibility condition at hanging nodes. The Lagrange multiplier test functions can be differently chosen, leading to different types of fine-tocoarse connection. Two main types of coupling are usually distinguished, i.e. strong and weak coupling.

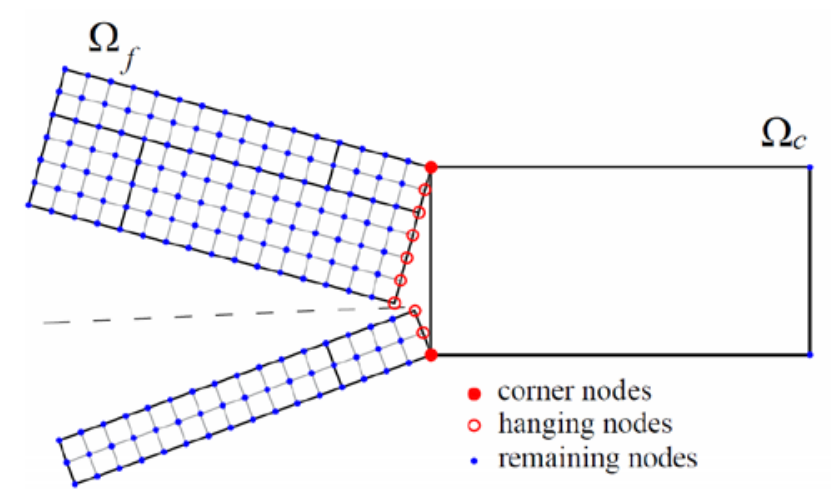

Fig. 6. Non-conforming mesh at the double-sided interface $\Gamma_{\text {int. }}$

In the strong coupling approach, the interface microscopic fluctuation is enforced to vanish pointwise by choosing for the Lagrange multiplier test function a linear combination of Dirac delta functions centered in all the nonmatching nodes belonging to the fine-scale side of $\Gamma_{\mathrm{int}}$; according to this approach, commonly referred to as collocation method, the interface compatibility conditions can be expressed via a set of linear multipoint constraint (see [47] for additional details). Conversely, in the weak coupling approach, also referred to as mortar approach, the interface microscopic fluctuation is enforced to vanish in average, based on a weak formulation for a variety of continuous test functions; such test functions are usually set as constants, coherently with the choice of linear shape functions for the displacement field, leading to a piecewise homogeneous distribution of interface tractions along $\Gamma_{\text {int. }}$

In this work, the strong coupling approach has been preferred to the weak one for two different reasons: (i) the use of a weak coupling technique increases the condition number of the assembled stiffness matrix, and ultimately, the number of iterations required by the Newton-Raphson solver to converge; and (ii) the strong coupling technique is the only approach to satisfy a priori the thermodynamic consistency conditions, since it does not allow damage percolation across the meso/macro interface.

\subsection{First failure surface for periodic masonry}


An important theoretical concept on which the adopted multiscale strategy relies is that of first failure surface, formulated for an infinite, rate-independent solid with a perfectly periodic microstructure, subjected to an arbitrary quasi-static macroscopic loading. Given the same repeating cell (RC) adopted for deriving the homogenized moduli of undamaged masonry, such a surface is defined as the (infinite) set of macroscopic strain states corresponding to the initiation of mesoscopic crack in the most stressed point of the cohesive interfaces inside this repeating cell. By considering a finite number of radial loading paths starting from the stress-free state, the first failure surface for a given masonry can be constructed, by interpolating the failure points obtained for all such paths.

After introducing a properly defined angular coordinate system $\left(\phi_{1}, \phi_{2}\right)$, any point representing a given macrostrain state can be expressed as follows:

$$
\begin{aligned}
& \varepsilon_{x}=\lambda \sin \phi_{1} \cos \phi_{2} \\
& \varepsilon_{y}=\lambda \sin \phi_{1} \sin \phi_{2}, \\
& \varepsilon_{x y}=\lambda \cos \phi_{1}
\end{aligned}
$$

$\lambda$ being the loading parameter controlling the magnitude of the imposed macrostrain. As the considered repeating cell is symmetric with respect the horizontal axis, only a half of the macrostrain space is constructed, i.e. the RC is subjected to the set of loading directions covering the ranges $0 \leq \phi_{1} \leq 90^{\circ}$ and $-180^{\circ} \leq \phi_{2} \leq 180^{\circ}$. The critical load factor $\lambda_{c}$ associated with each macrostrain path direction can be obtained in the post-processing step, by virtue of linearity of the overall response of the microstructure up to this value, as $\lambda_{c}=\delta_{0} / \delta_{\text {eq,max }}^{1}, \delta_{0}$ being the critical displacement jump defined in Section 2.1 and $\delta_{\text {eq,max }}^{1}$ the equivalent displacement jump per unit $\lambda$ at the most stressed point of the cohesive interfaces.

The first failure surface (also referred to as elastic limit surface) is discretized here by using four-node bilinear quadrilateral shaped elements, as shown in Fig. 7, in a similar manner than adopted in [44]. As such a geometric locus is expected to be of a non-smooth type, a sufficiently fine discretization must be performed, in order to accurately determine the critical load factor $\lambda_{c}$, even in the neighborhood of discontinuities; such a fine discretization is achieved by considering a sufficiently high number of macrostrain path directions, i.e. by choosing a very small angular increment $\Delta \phi=\Delta \phi_{1}=\Delta \phi_{2}$.

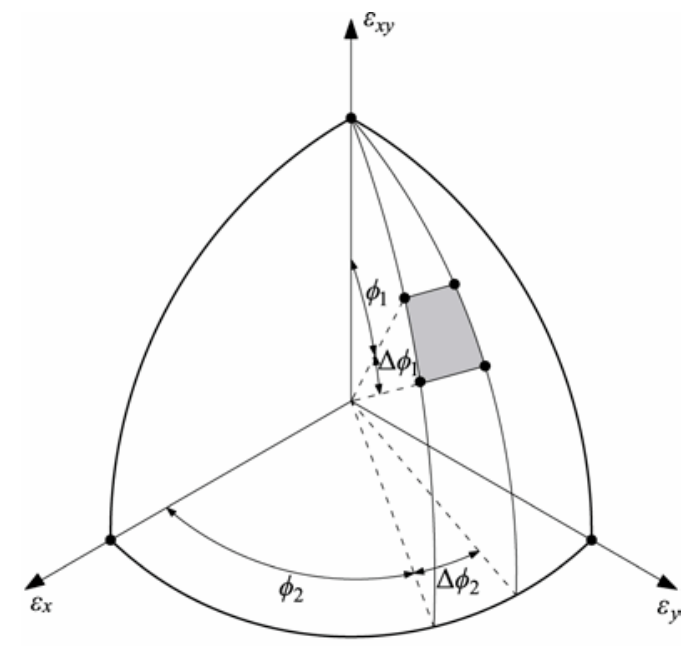

Fig. 7. Discretized first failure surface in the macrostrain space; each nodal value is numerically generated by solving a BVP on the adopted repeating cell assuming plane stress and periodic boundary conditions.

\section{Description of the adaptive model refinement technique}


In this section, the proposed strategy for the multiscale failure analysis of periodic masonry is described, together with its computational details within the finite element setting. Such a strategy belongs to the wide group of adaptive model refinement approaches; analogous to the most used adaptive mesh refinement, the adaptive model refinement strategy consists in replacing the homogenized macroscopic coarse-scale model by the original fine-scale model in the model subdomains for which a certain switching criterion is satisfied, in an automatic manner during damaged evolution. At the beginning of simulation, the entire model domain is partitioned in a finite number of coarse-level finite elements (referred to as macro-elements) arranged in a rectangular array, coherently with the shape of the chosen repeating cell, as shown in Fig. 8a; after that, the macro-elements for which the switching threshold is reached are flagged as critical, and replaced by (finely meshed) repeating cells (see Fig. 8b); such a model refinement is repeated within an incremental-iterative strategy until the final collapse is reached (see Fig. 8c).

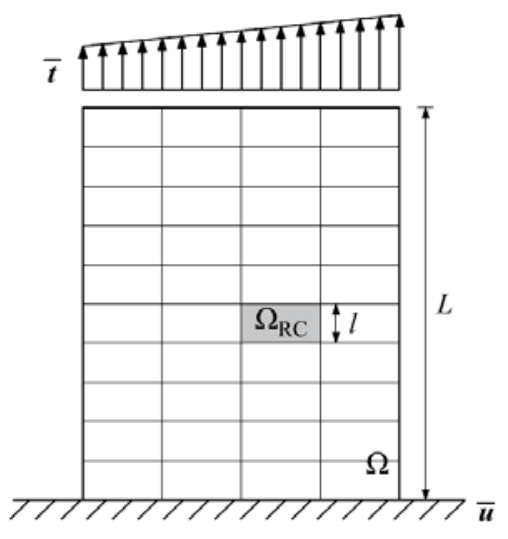

(a)

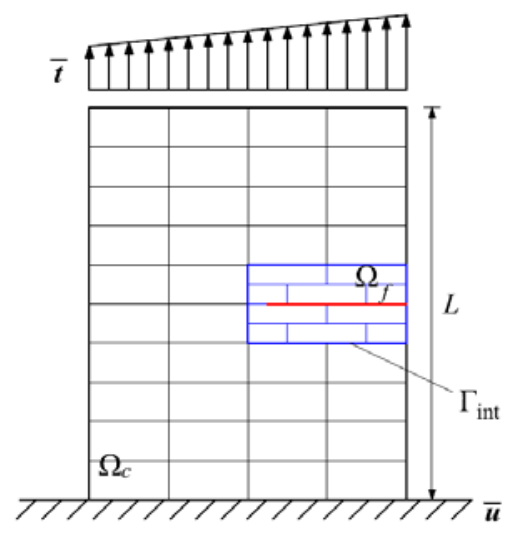

(b)

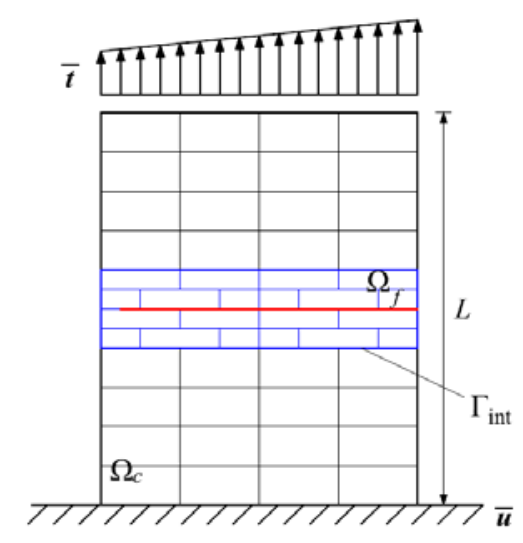

(c)

Fig. 8. Three stages within the adaptive model refinement strategy: (a) initial stage, for which the given microstructure is replaced everywhere by an equivalent homogenized material; (b) intermediate stage, at which the masonry structure is partially damaged; (c) final stage, at incipient collapse.

The above-mentioned model refinement necessarily requires the introduction of a switching criterion, which is able to automatically detect the critical macro-elements as the ones characterized by the appearance of the first nonlinearities. The proposed criterion, which is mainly based on the construction of the first failure surface described in Section 2.4, is a refined version of the twofold criterion proposed in the authors' previous work [50]. Such a criterion was developed to take into account the fact that first-order homogenization techniques are not rigorously valid in the regions affected by notable strain gradient; therefore, as better explained in [50], the model refinement is performed not only for macro-elements whose strain state is outside the first-failure locus, but also for those characterized by sufficiently high macrostrain gradients, regardless of the macrostrain level. The main drawback of this criterion is that it potentially leads to extended zoomed-in areas (i.e. those characterized by non-negligible higher-order macrostrain terms according to the given threshold) although it would not be absolutely needed, and thus reducing the overall computational efficiency.

On the contrary, the newly proposed criterion, which is regarded as optimal in terms of both accuracy and computational cost, is based on the determination of a modified first failure domain for macroscale regions characterized by sufficiently high macrostrain gradients; in particular, it is assumed that the actual first failure locus cannot be directly used for macro-elements exhibiting high hourglass energy contents, i.e. for which $\phi_{\text {hourglass }} \geq \alpha_{c} \phi, \phi$ being the total internal energy, $\phi_{\text {hourglass }}$ the hourglass energy and $\alpha_{c}$ the given threshold (see [50] for additional details). As a consequence, in such a case, a reduced failure domain has been considered, which is heuristically derived, by homothetically shrinking the actual one via the arbitrarily chosen scale factor $c$. In the proposed adaptive model refinement, the numerical parameters $\alpha_{c}$ and $c$ have to 
be calibrated within the modeling process, in order to reach an optimal compromise between efficiency and accuracy for the multiscale analysis. The related numerical algorithm, sketched in Table 1, is based on a predictor-corrector strategy, according to which, after new microscale subdomains are created or existing microscale subdomains are enlarged, the equilibrium state is recomputed for the current load step. As a consequence of the adaptive model refinement, the damage variable must be transferred from the old to the new mesh and the global stiffness matrix must be recomputed at each iteration.

It is worth noting that this multiscale algorithm can be regarded as a generalization of both algorithms proposed in [50] and there referred to as algorithms A1 and A2; indeed, for $c=1$, the new algorithm turns to be coincident with algorithm A1, whereas when $c$ goes to zero, the equivalence with algorithm A2 is recovered.

Table 1. Proposed incremental-iterative method for the multiscale failure analysis of masonry.

\section{START}

0. Read the input data: geometry, mesh, material properties, boundary conditions, damage variable, etc.

1. Initialize the nodal displacement vector, $\boldsymbol{u}=\mathbf{0}$

2. Loop for model refinement iteration

2.1. Loop for load increment

2.1.1. Determine the external force vector $\boldsymbol{f}_{\mathrm{ext}}$

2.1.2. Loop for Newton-Raphson iteration

2.1.2.1. Compute the tangent stiffness matrix $\boldsymbol{K}_{t}$ and the internal force vector $\boldsymbol{f}_{\text {int }}$

2.1.2.2. Solve the linear system $\boldsymbol{K}_{t} \Delta \boldsymbol{u}=\boldsymbol{f}_{\text {ext }}-\boldsymbol{f}_{\text {int }}$ for $\Delta \boldsymbol{u}$

2.1.2.3. Update the nodal displacement vector, $\boldsymbol{u}=\boldsymbol{u}+\Delta \boldsymbol{u}$

2.1.2.4. Update the damage variable

2.1.2.5. Check the convergence; if the convergence has not occurred, go back to step 2.1.2

2.1.3. Compute stress and strains for each element

2.1.4. Loop for macro-element

2.1.4.1. Compute the hourglass energy ratio $\phi_{\text {hourglass }} / \phi$; if the hourglass ratio is greater than $\alpha_{c}$, shrink the first failure domain by the scale factor $c$

2.1.4.2. Compute the macrostrain via integration of boundary displacements; if the macrostrain point is outside the first failure domain, flag the macro-element as critical

2.1.5. If any critical macro-element is detected, perform model refinement, transfer the damage variable to the new mesh and go back to step 2

END

2.1.6. Update the load increment counter; if the analysis is not complete, go back to step 2.1

The adaptive multiscale method has been implemented within the commercial finite element software COMSOL Multiphysics ${ }^{\circledR}$ [59], by taking advantage of its extended scripting capabilities in MATLAB ${ }^{\circledR}$ language. The built-in continuation solver has been used, equipped with a suitably defined stop condition implementing the proposed zooming-in criterion. The outer model refinement loop is performed via a dedicated MATLAB ${ }^{\circledR}$ code linked to the main computational environment, which is responsible for the inner Newton-Raphson loop.

\section{Application to masonry with traditional mortar joints: the shear wall test}

In this section, the efficacy of the proposed multiscale strategy has been demonstrated for the failure analysis of a masonry wall with traditional mortar joints and subjected to the shear test introduced in [60]; such a test has been chosen as an example of masonry subjected to very complex cracking, whose location and geometric pattern are not known a priori neither easily predictable by engineering intuition. In particular, the 
considered wall exhibits a shear-flexure behavior under the action of in-plane lateral loads, which is typical for real-life masonry structures under seismic events.

\subsection{Material and geometric properties}

The considered test specimen is a small-sized wall with dimensions $980 \times 1106 \mathrm{~mm}^{2}$, resulting in a width/height ratio of about one; such a wall presents a central opening of $220 \times 372 \mathrm{~mm}^{2}$, as shown in Fig. 9 . An initial confinement is applied by prescribing a uniform vertical displacement of the wall top until a mean compressive stress of $0.30 \mathrm{MPa}$ is reached. After that, a top horizontal load is monotonically increased under displacement control in a confined way, i.e. with constrained rotation and vertical displacement of the wall top.

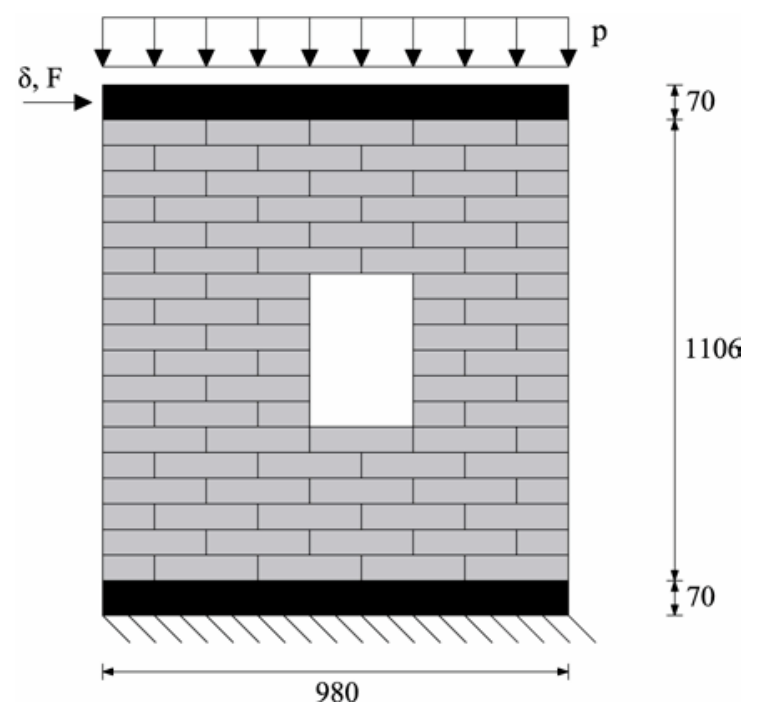

Fig. 9. Masonry wall with opening subjected to a shear test: geometry and boundary conditions (all dimensions are expressed in millimeters).

This wall is made of solid clay bricks of $210 \times 52 \times 100 \mathrm{~mm}^{3}$ in dimension, separated by mortar joints of 10 $\mathrm{mm}$ in thickness. The elastic behavior of brick material is assumed to be linearly isotropic, and characterized by the material constants $E_{b}=16,000 \mathrm{MPa}$ and $v_{b}=0.15$, whereas the elastic behavior of mortar joints, which are initially undamaged, is defined by means of the normal and tangential stiffness components, i.e. $K_{n}$ and $K_{s}$, obtained via Eq. (4), which in turn depend on the material constants of both mortar and bricks; the elastic constants of mortar are $E_{m}=782 \mathrm{MPa}$ and $v_{m}=0.14$. For this test, in order to reduce the associated computational cost, bricks are undamageable due to the assumed relatively low strength of mortar joints, and thus the only source of nonlinearity relies on the inelastic behavior of mortar joints, according the cohesive traction-separation law discussed in Section 2.1. The required inelastic parameters of mortar, i.e. the uniaxial tensile, uniaxial compressive and biaxial compressive strengths, denoted by $\sigma_{t}, \sigma_{c}$ and $\sigma_{b}$, respectively, as well as the mode-I fracture energy $G_{f}$, are listed in Table 2; the latter quantity is obtained from the fracture energy per unit volume of mortar, accepting that damage is allowed to spread on the entire joint thickness (see [57]).

Table 2. Inelastic material parameters of mortar joints.

\begin{tabular}{|c|c|c|c|}
\hline$\sigma_{t}\left[\mathrm{~N} / \mathrm{mm}^{2}\right]$ & $\sigma_{c}\left[\mathrm{~N} / \mathrm{mm}^{2}\right]$ & $\sigma_{b}\left[\mathrm{~N} / \mathrm{mm}^{2}\right]$ & $G_{f}[\mathrm{~N} / \mathrm{mm}]$ \\
\hline 0.25 & 10.5 & 12.6 & 0.18 \\
\hline
\end{tabular}




\subsection{Multiscale numerical simulation (MNS) results}

As a preliminary step for the multiscale numerical simulation (MNS), both the homogenized moduli and the first failure surface for the given masonry has been numerically determined assuming a plane stress state and periodic boundary conditions, according to the procedures described in Sections 2.2 and 2.4, respectively.

The overall moduli, numerically obtained for the adopted repeating cell by using a sufficiently refined mapped mesh whose size is about $4.5 \mathrm{~mm}$ (see Fig. 10), are listed in Table 3. As expected, the moduli $C_{k k 12}^{\text {hom }}$ have been found to be null, confirming the well-known orthotropic nature of undamaged masonry.

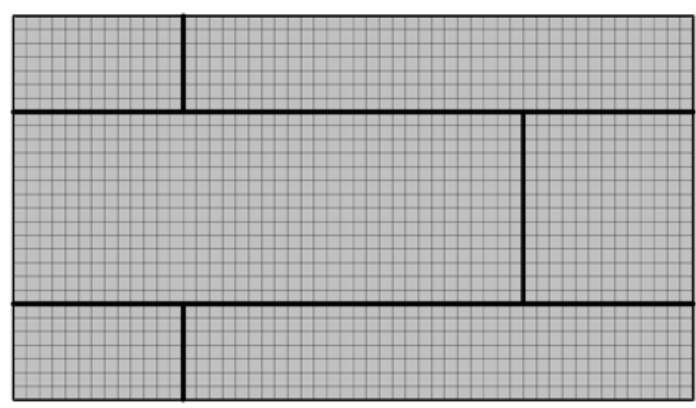

Fig. 10. Adopted mesh for the considered RC of the wall.

Table 3. Homogenized moduli for the in-plane response of the given masonry microstructure.

\begin{tabular}{|r|r|r|r|r|r|}
\hline$C_{1111}^{\text {hom }}[\mathrm{GPa}]$ & $C_{1122}^{\text {hom }}[\mathrm{GPa}]$ & $C_{112}^{\text {hom }}[\mathrm{GPa}]$ & $C_{2222}^{\text {hom }}[\mathrm{GPa}]$ & $C_{2212}^{\text {hom }}[\mathrm{GPa}]$ & $C_{1212}^{\text {hom }}[\mathrm{GPa}]$ \\
\hline 9.830 & 0.344 & 0 & 3.911 & 0 & 1.524 \\
\hline
\end{tabular}

Using the same repeating cell and the same mesh as for the determination of the homogenized moduli, several (linear) mesoscopic problem have been solved by varying the macrostrain direction $\left(\phi_{1}, \phi_{2}\right)$ in order to derive the relative numerical values of the critical load factor $\lambda_{c}$, needed for the determination of the first failure surface. Such a locus, defined on the macrostrain space and whose shape has not been assumed a priori by phenomenological arguments, has been found to be a multi-surface damage locus.

This locus can be effectively represented as a contour graph defined on the principal macrostrain plane ( $\varepsilon_{1}-\varepsilon_{2}$ plane); accordingly, a one-parameter family of closed curves have been numerically determined by varying the angle $\theta$, ranging from $0^{\circ}$ to $90^{\circ}$, which defines the orientation of the principal macrostrain system with respect to the orthotropic axes. For the sake of clarity, only seven contour lines have been reported in Fig. 11, referring to the angular values from $0^{\circ}$ to $90^{\circ}$. It is worth noting that the contour lines in the range $45^{\circ} \leq \theta \leq 90^{\circ}$ have been directly deduced by swapping $\varepsilon_{1}$ and $\varepsilon_{2}$ for the contour lines referring to complementary angles. 


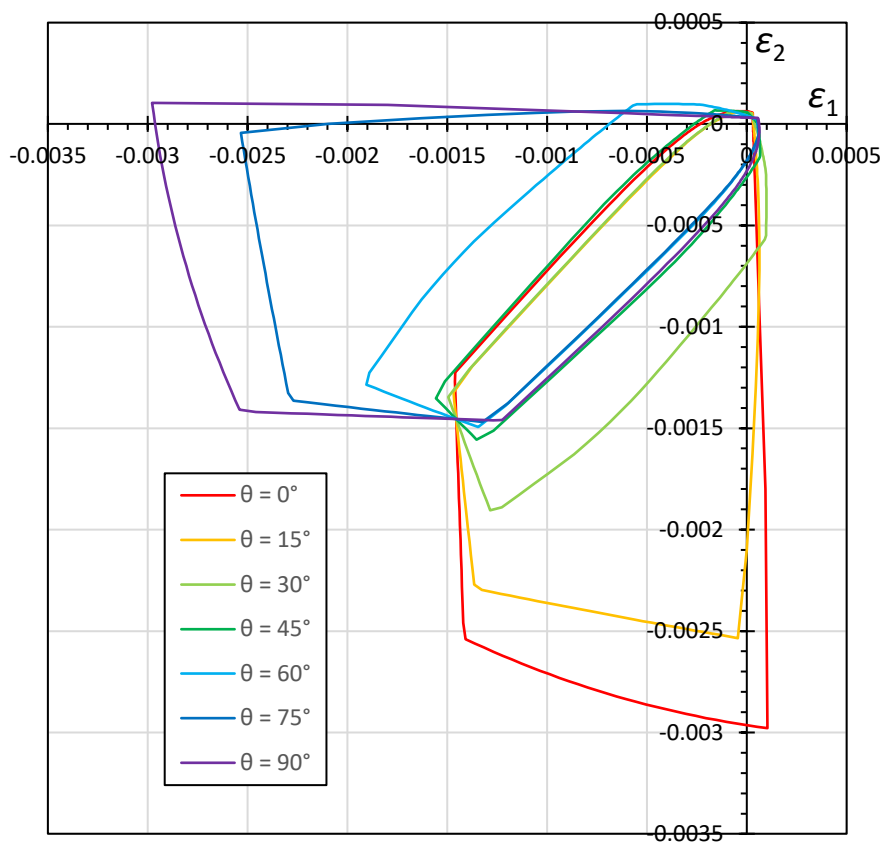

Fig. 11. Microscopically informed first failure locus for periodic masonry with traditional mortar joints, represented as a one-parameter family of contour lines in the principal macrostrain plane.

The previously determined first failure locus does not take into account any damage mechanism inside the bricks, coherently with the assumed relatively low strength of mortar joints; this choice is motivated by the need of reducing the overall computational cost of the subsequent multiscale numerical simulation (MNS). It is worth noting that this assumption, which can be believed to hold for old mortar joints, is no longer valid for fiber-reinforced mortar joints, as will be shown in Section 5.2.

All the numerical results reported in this section have been determined by using the proposed adaptive multiscale strategy, according to the incremental-iterative method sketched in Table 1. Fig. 12 shows the multiscale structural response for the shear wall test obtained in terms of load-displacement curve; in order to explore the role of the free numerical parameters of the proposed multiscale method on both accuracy and efficiency, a double parametric analysis has been carried out by independently varying $\alpha_{c}$ and $c$. For the considered numerical experiment, $\alpha_{c}$ has been varied in the range between 0.05 and 0.20 , whereas $c$ has been chosen in the range between $1 / 1.5$ and $1 / 5$. It can be noted that the choice of such parameters exerts a limited influence on the predicted overall structural response; this is in line with the resulting crack pattern, which is globally independent of the adopted values of $\alpha_{c}$ and $c$, as shown by the damage variable map reported in Fig. 13.

By analyzing more closely the structural responses of Fig. 12, a number of discontinuity points can be identified, which may be attributed to the combination of both homogenization and coupling errors, which cannot be controlled during the adaptive model refinement step by using the proposed multiscale algorithm. These errors inevitably cause a non-negligible delay for the nucleation and propagation of damage in the neighborhood of the meso-to-macro interface and may lead to the loss of validity of the numerical results. It follows that, in order to assess the proposed multiscale methodology, the following a posteriori error is introduced:

$$
e_{F}:=\frac{F_{\text {pred }}-F_{\text {corr }}}{F_{\text {pred }}} \times 100,
$$

defined as the percent variation in the applied force before $\left(F_{\text {pred }}\right)$ and after $\left(F_{\text {corr }}\right)$ the iterative correction within each load incremental step. The major error values attained during the loading history, reported in Table 4 for the all considered numerical simulations, are all in the range between $3 \%$ and $10 \%$, meaning that the choice of the above-mentioned numerical parameters exerts a limited influence on the accuracy of the 
multiscale method; the accuracy of the multiscale method is very high in the considered range for $\alpha_{c}$ and $c$, as confirmed by the small average value of $e_{F}$, found to be less than $6 \%$. Moreover, as expected, these errors tend to decrease as the parameter $c$ is reduced; this is due to the fact that the zooming-in step is anticipated, allowing the existing cracks to propagate without experiencing any artificial toughening effects associated with the proximity of a meso-to-macro interface.
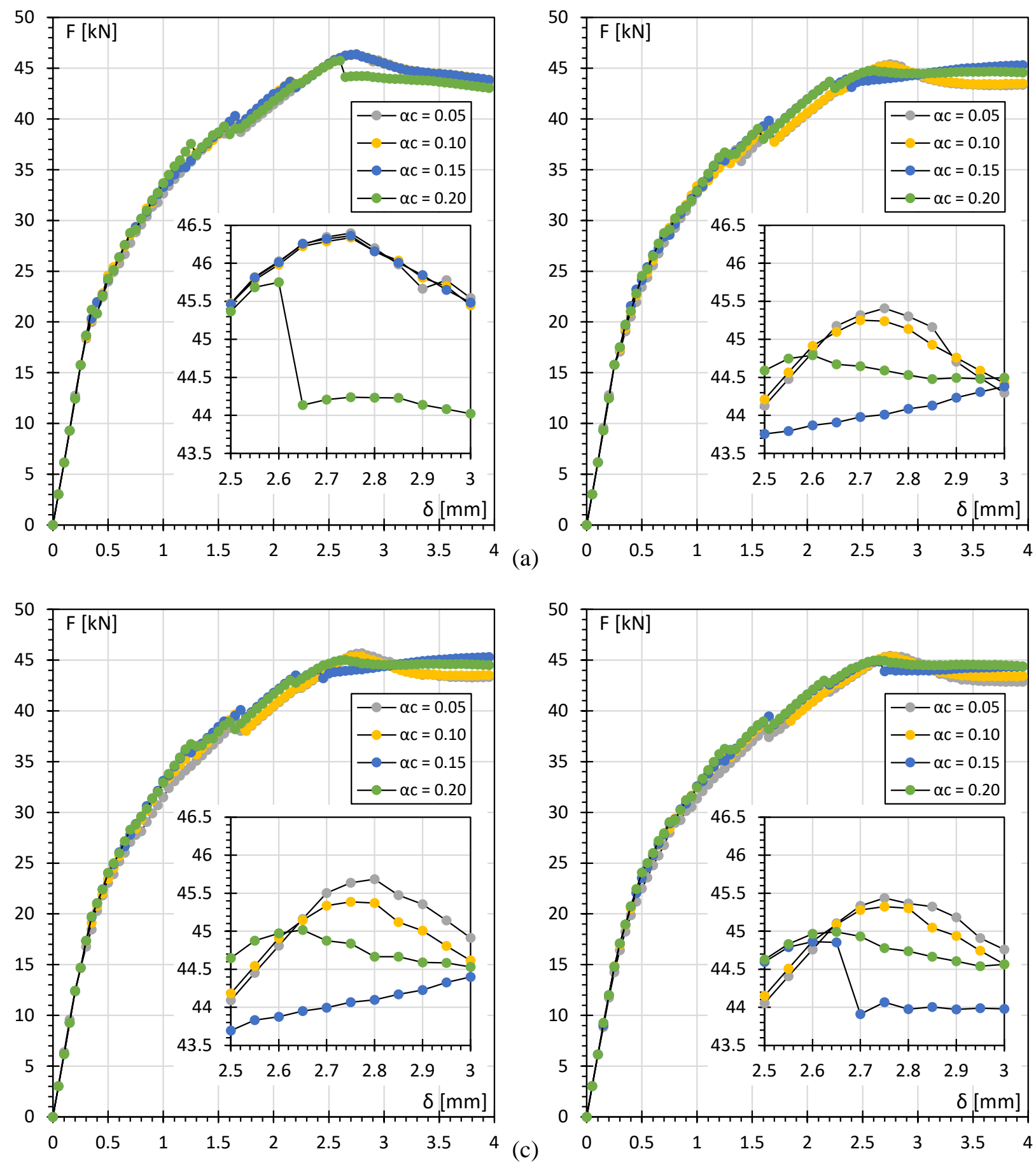

Fig. 12. Force versus displacement curve obtained from the multiscale numerical simulation (MNS) performed according to the proposed algorithm, for different values of both the tolerance parameter $\alpha_{c}$ and the scale factor $c$ : (a) $c=1 / 1.5$; (b) $c=1 / 2$; (c) $c=1 / 3$; (d) $c=1 / 5$. 

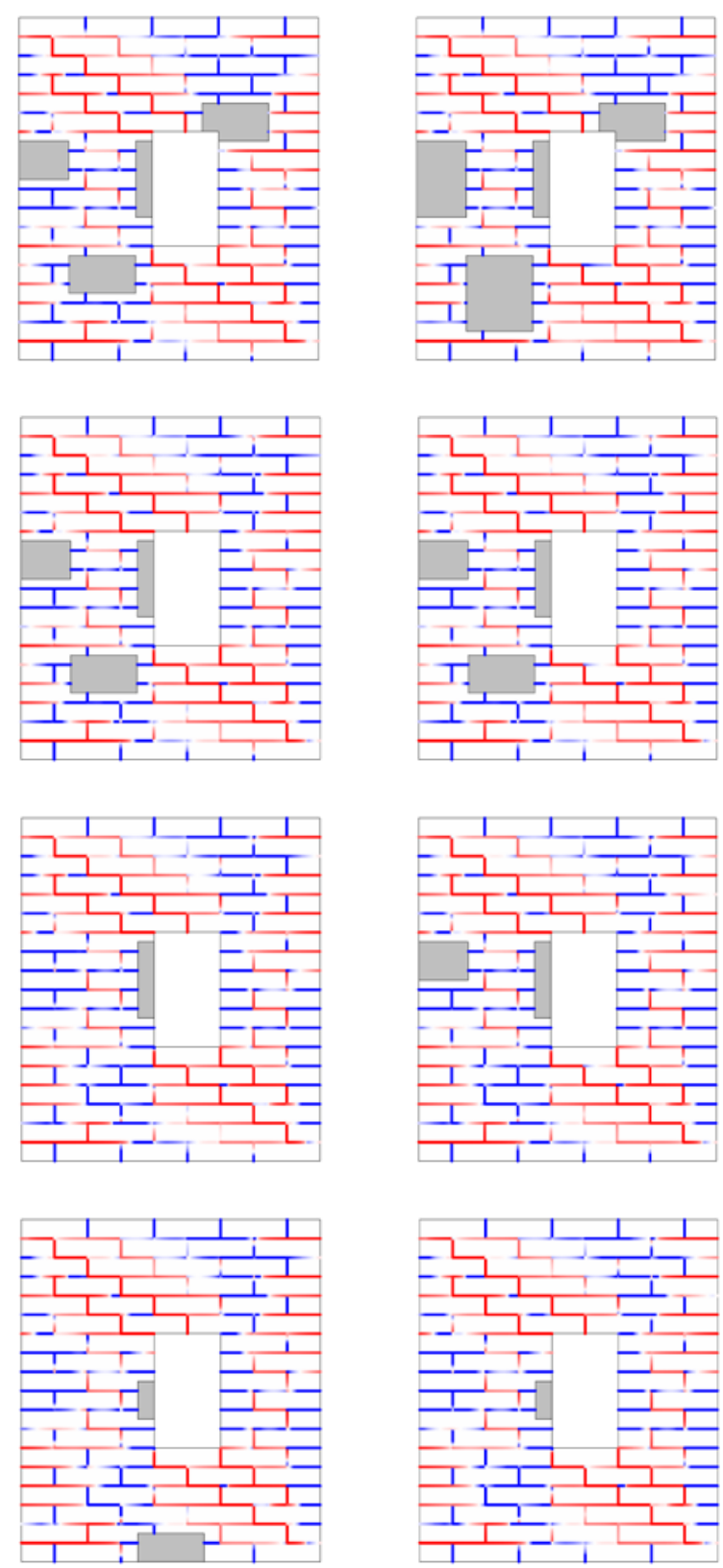

$\alpha_{c}=0.05$
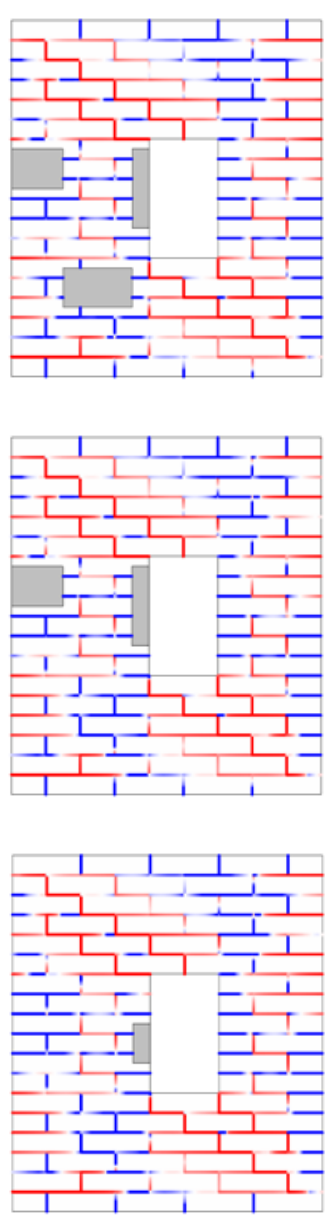

$\alpha_{\mathrm{c}}=0.10$
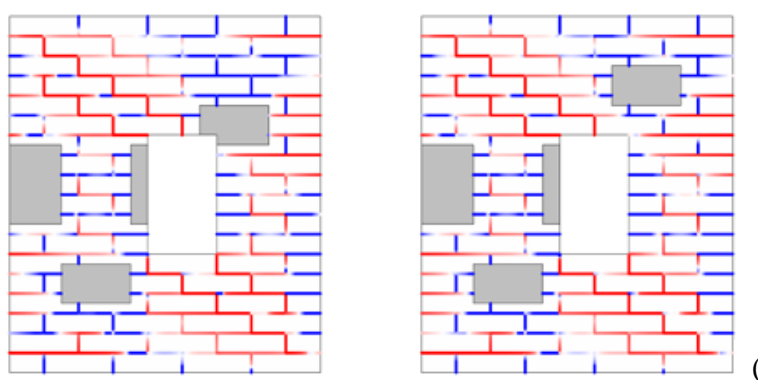

(a)
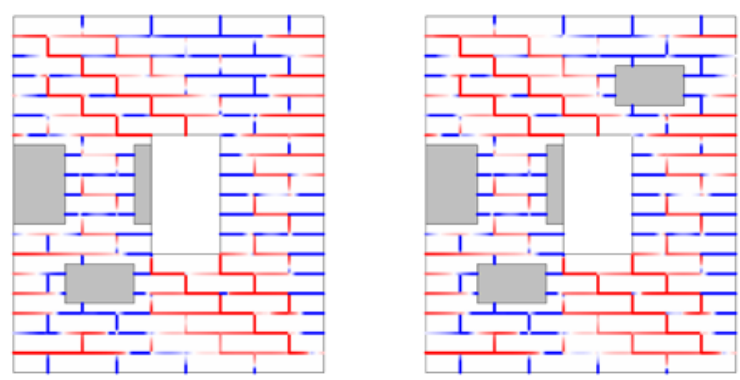

(b)
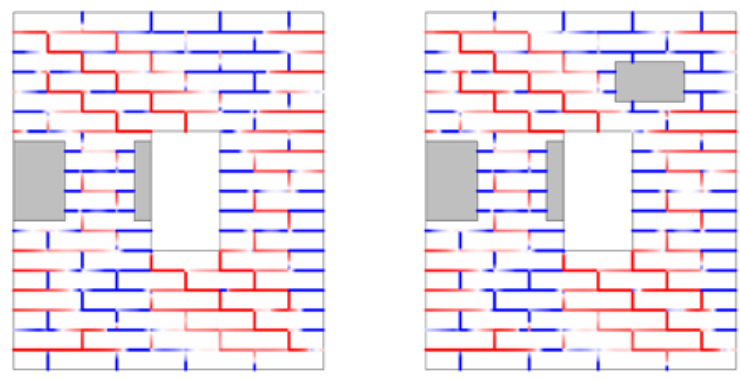

(c)
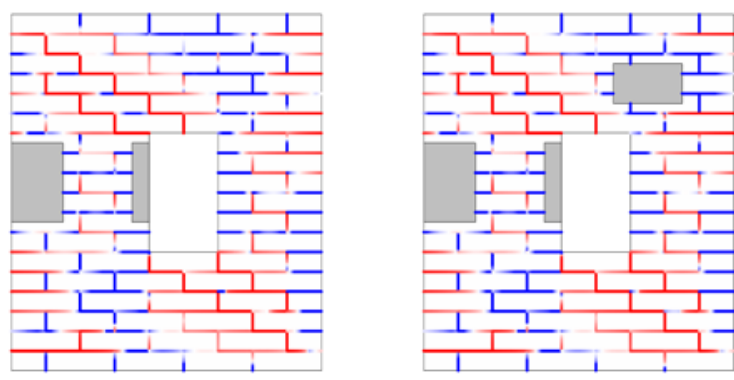

(d)

$\alpha_{\mathrm{C}}=0.15$

Fig. 13. Damage variable maps for $\delta=4.0 \mathrm{~mm}$ obtained from the multiscale numerical simulation (MNS) performed according to the proposed algorithm, for different values of both the tolerance parameter $\alpha_{c}$ and the scale factor $c$ : (a) $c=1 / 1.5$; (b) $c=1 / 2$; (c) $c=1 / 3$; (d) $c=1 / 5$; the coarse-level elements outside the zone of interest are shaded in grey, whereas the damage variable defined on the cohesive interfaces ranges from 0 (blue) to 1 (red).

Table 4. Estimation of the a posteriori error $e_{F}$ (expressed as a percent) for assessing the validity of the present multiscale numerical simulations (MNSs).

\begin{tabular}{|c|c|c|c|c|c|}
\hline \multicolumn{2}{|c|}{} & \multicolumn{4}{|c|}{$c$} \\
\cline { 3 - 6 } \multicolumn{2}{c|}{} & $1 / 1.5$ & $1 / 2$ & $1 / 3$ & $1 / 5$ \\
\hline \multirow{4}{*}{$\alpha_{c}$} & 0.05 & 5.38 & 9.01 & 3.43 & 4.24 \\
\cline { 2 - 6 } & 0.10 & 5.06 & 9.11 & 5.68 & 4.46 \\
\cline { 2 - 6 } & 0.15 & 5.78 & 8.08 & 5.68 & 3.41 \\
\cline { 2 - 6 } & 0.20 & 9.39 & 8.08 & 5.68 & 3.30 \\
\hline
\end{tabular}


On the other hand, the choice of parameters $\alpha_{c}$ and $c$ has a strong influence on the overall efficiency of the multiscale method; this is essentially due to the very different extensions of the fine-scale region detected at the early stages of simulation by using different values of these parameters, as clearly shown in Fig. 14, reporting the related damage maps referring to the incremental step $\delta=0.25 \mathrm{~mm}$.
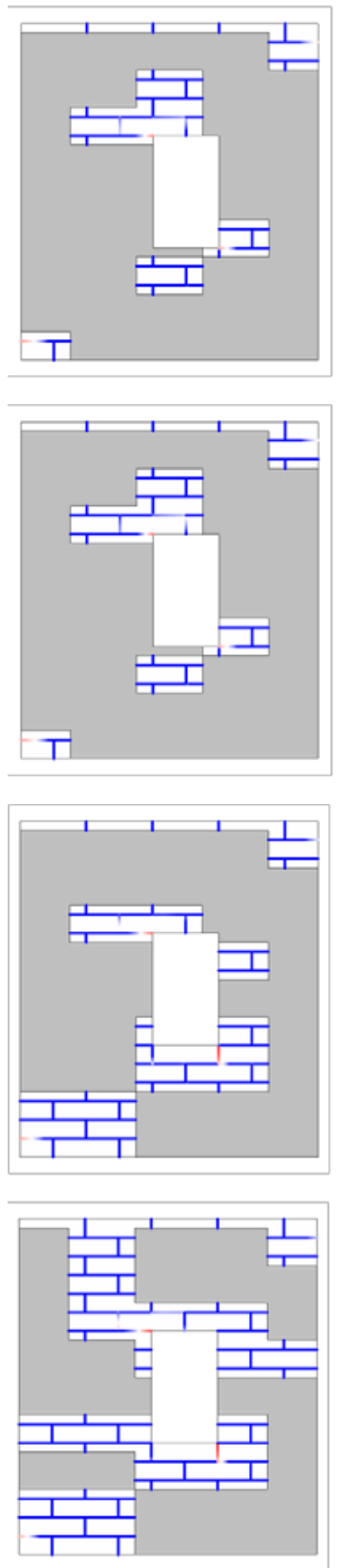

$\alpha_{c}=0.05$
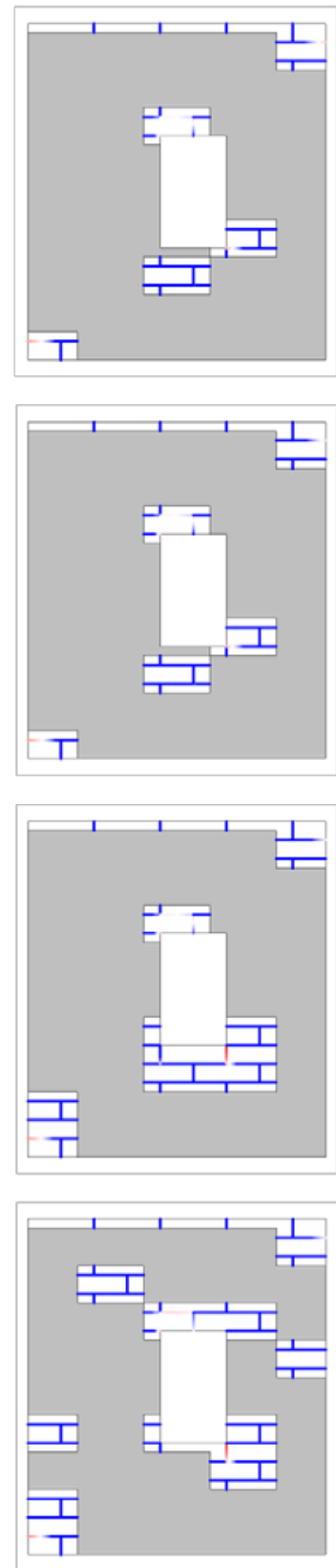

$\alpha_{c}=0.10$
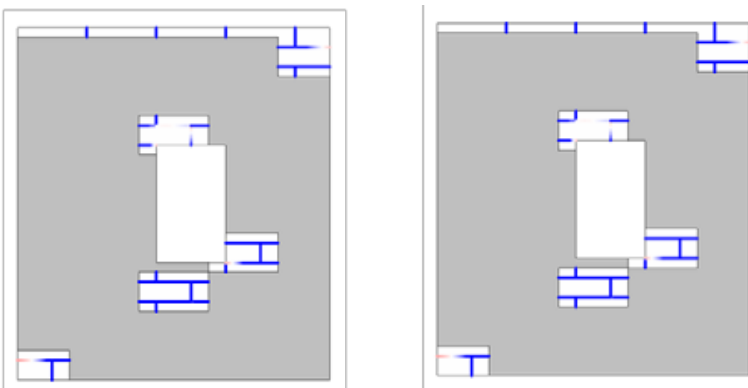

(a)
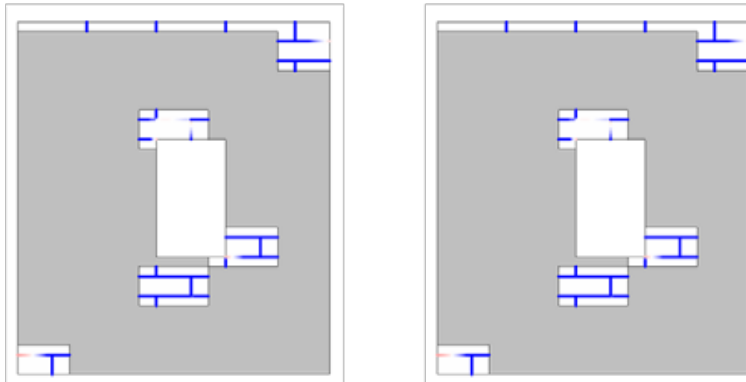

(b)
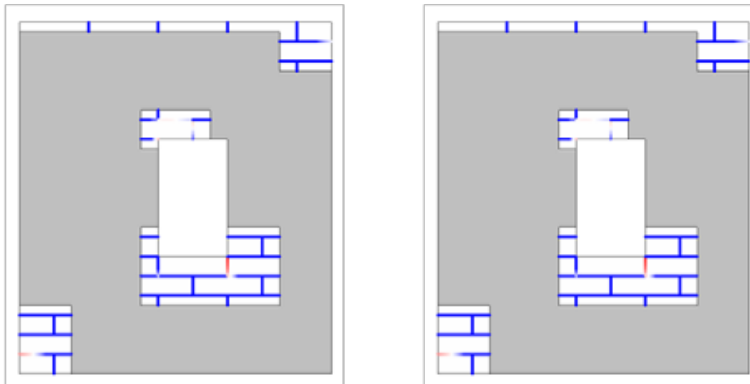

(c)

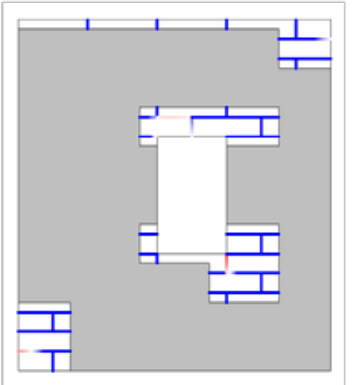

$\alpha_{c}=0.15$

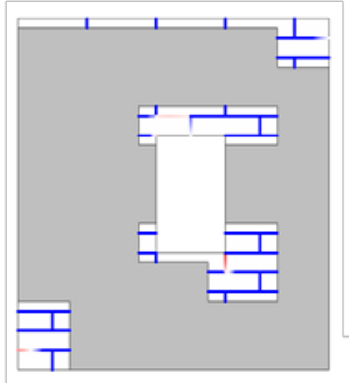

(d)

Fig. 14. Damage variable maps for $\delta=0.25 \mathrm{~mm}$ obtained from the multiscale numerical simulation (MNS) performed according to the proposed algorithm, for different values of both the tolerance parameter $\alpha_{c}$ and the scale factor $c$ : (a) $c=1 / 1.5$; (b) $c=1 / 2$; (c) $c=1 / 3$; (d) $c=1 / 5$; the coarse-level elements outside the zone of interest are shaded in grey, whereas the damage variable defined on the cohesive interfaces ranges from 0 (blue) to 1 (red).

By analyzing Fig. 14, it can be noted that the computational efficiency of the proposed multiscale method can be improved by increasing either $\alpha_{c}$ or $c$, resulting in a dramatic reduction in the extension of the zoomed-in region at the early stages of simulation; tuning both of them, it can be achieved the optimal 
balance between accuracy and efficiency. Furthermore, for $c$ approaching 1 , the influence of the critical hourglass energy ratio $\alpha_{c}$ on the computational efficiency at the early stages of simulation becomes negligible. Conversely, if $c$ tends to 0 , the efficiency of the multiscale is strongly affected by the choice of $\alpha_{c}$; if a very low value of such a parameter is selected, the size of the zone of interest is very large with respect to the entire computational domain; it means that the zooming-in operations are anticipated with respect to the appearance of nonlinearities in the constitutive response. In the limit case, for $c=0$ and $\alpha_{c}=0$, the model refinement steps are performed regardless of both the stress level and the hourglass energy content for any macro-element.

A deeper insight into the overall efficiency of the proposed multiscale method is presented in Section 4.3, where a detailed analysis of the related computational performances is performed, by varying both $c$ and $\alpha_{c}$ within the considered numerical ranges.

\subsection{Validation with experiments and alternative multiscale numerical simulation results}

In order to illustrate the effectiveness of the multiscale numerical simulation (MNS) presented in Section 4.2, the related results are suitably compared with solutions obtained by a direct numerical simulation (DNS) and alternative multiscale numerical simulations as well as with some experimental data found in the literature.

Fig. 15 shows a comparison in terms of force versus displacement curve between the multiscale numerical simulations (MNSs) performed by using the present algorithm (referred to as algorithm A3) and those proposed in [50] (referred to as algorithms A1 and A2), the direct numerical simulation (DNS), and the experimental results presented in [60]. It is worth remind here that algorithm A1 does not require any parameter, whereas algorithm A2 introduces only the parameter $\alpha_{c}$ (see [50] for additional details); the tested values for $\alpha_{c}$ are the same as those adopted for algorithm A3. The structural responses numerically obtained by using the multiscale algorithms A2 and A3 have been reported as envelopes of families of curves referring to the tested values for the involved parameters ( $\alpha_{c}$ for algorithm A2, $\alpha_{c}$ and $c$ for algorithm A3).

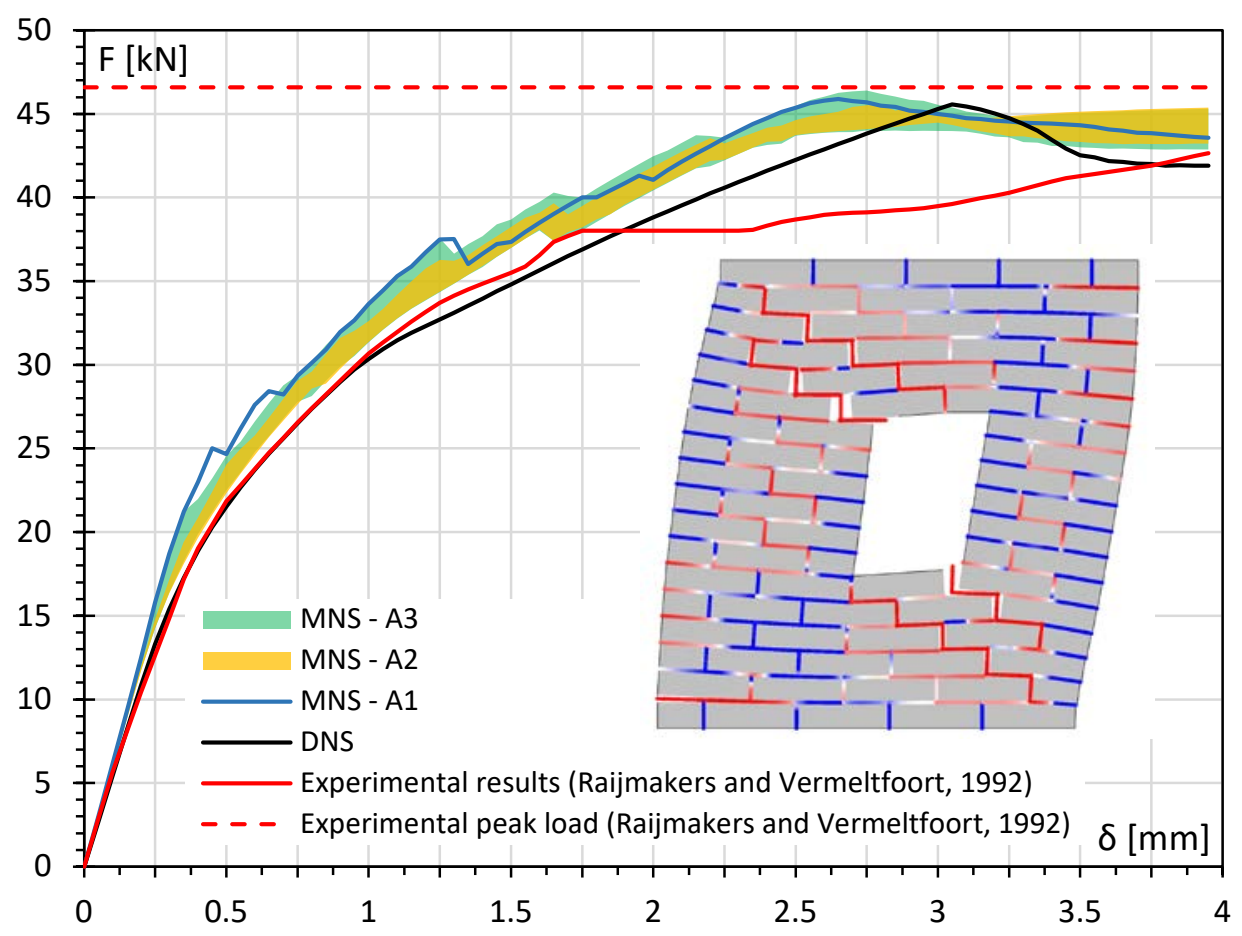

Fig. 15. Comparison in terms of force versus displacement curve between the multiscale numerical simulations (MNSs) performed by using the present algorithm (referred to as algorithm A3) and those proposed in [50] (referred to as algorithms A1 and A2), the direct numerical simulation (DNS), and the experiments by Raijmakers and Vermeltfoort [60]. 
It can be observed that all the considered multiscale algorithms may lead to a good prediction of the overall structural response; in particular, the peak load obtained by means of algorithms A1, A2 or A3 is very close to both the experimental peak load and that numerically predicted by a direct numerical simulation, here regarded as the reference solution. The main advantage of the proposed multiscale strategy (i.e. algorithm A3) over the previously proposed algorithms A1 and A2 relies in the possibility of a fine tuning of its numerical parameters, which ultimately leads to achieve a suitable balance between desired accuracy and available computational resources.

Table 5 shows the same comparisons as Fig. 15 in terms of both peak load and initial stiffness, together with the relevant percentage errors; all the considered multiscale algorithms provide very small percentage errors with respect the reference solution (i.e. the direct numerical simulation), ranging between $0.05 \%$ and $1.76 \%$; the smallest error is exhibited by the present multiscale algorithm (i.e. A3) for the case $c=1 / 3$ and $\alpha_{c}=0.05$, confirming that a suitable tuning of the free numerical parameters leads to achieve a better accuracy than the other algorithms, referred to as A1 and A2, previously proposed by the same authors in [50]; moreover, the error on the peak load has an overall tendency to increase as $\alpha_{c}$ increases, for any value of $c$. Conversely, the error on the initial stiffness is almost independent of the tolerance factor, remaining systematically overestimated of about $13 \%$. Such an error is not related to the adopted zooming-in technique, but essentially represents a homogenization error which relies on the assumed perfect periodicity for the determination of the overall moduli of masonry material (in the linear range), even in the neighborhood of boundary layers, where such conditions cease to hold.

Table 5. Comparisons in terms of predicted peak load and initial stiffness between the multiscale numerical simulations (MNSs) performed by using the present algorithm and those proposed in [50] (referred to as algorithms A1 and A2), and the direct numerical simulation (DNS).

\begin{tabular}{|c|c|c|c|c|c|c|}
\hline & \multicolumn{2}{|l|}{ Peak load } & \multicolumn{2}{|l|}{ Initial stiffness } \\
\hline & & & $F_{\max }[\mathrm{kN}]$ & Error [\%] & $K_{0}[\mathrm{kN} / \mathrm{mm}]$ & Error [\%] \\
\hline \multicolumn{3}{|c|}{ DNS (reference) } & 45.561 & & 53.702 & \\
\hline \multirow{16}{*}{ MNS (present) } & \multirow{4}{*}{$c=1 / 1.5$} & $\alpha_{c}=0.05$ & 45.936 & 0.823 & 60.382 & 12.44 \\
\hline & & $\alpha_{c}=0.10$ & 46.336 & 1.701 & 60.382 & 12.44 \\
\hline & & $\alpha_{c}=0.15$ & 46.362 & 1.758 & 60.382 & 12.44 \\
\hline & & $\alpha_{c}=0.20$ & 45.751 & 0.417 & 60.382 & 12.44 \\
\hline & \multirow{4}{*}{$c=1 / 2$} & $\alpha_{c}=0.05$ & 45.410 & -0.331 & 60.382 & 12.44 \\
\hline & & $\alpha_{c}=0.10$ & 45.253 & -0.676 & 60.382 & 12.44 \\
\hline & & $\alpha_{c}=0.15$ & 45.306 & -0.560 & 60.382 & 12.44 \\
\hline & & $\alpha_{c}=0.20$ & 44.791 & -1.690 & 60.382 & 12.44 \\
\hline & \multirow{4}{*}{$c=1 / 3$} & $\alpha_{c}=0.05$ & 45.585 & 0.0537 & 60.382 & 12.44 \\
\hline & & $\alpha_{c}=0.10$ & 45.387 & -0.382 & 60.382 & 12.44 \\
\hline & & $\alpha_{c}=0.15$ & 45.302 & -0.568 & 60.382 & 12.44 \\
\hline & & $\alpha_{c}=0.20$ & 45.017 & -1.194 & 60.382 & 12.44 \\
\hline & \multirow{4}{*}{$c=1 / 5$} & $\alpha_{c}=0.05$ & 45.437 & -0.272 & 61.056 & 13.69 \\
\hline & & $\alpha_{c}=0.10$ & 45.326 & -0.516 & 60.972 & 13.54 \\
\hline & & $\alpha_{c}=0.15$ & 44.863 & -1.532 & 60.537 & 12.73 \\
\hline & & $\alpha_{c}=0.20$ & 44.990 & -1.253 & 60.537 & 12.73 \\
\hline \multicolumn{3}{|l|}{ MNS (A1) } & 45.894 & 0.731 & 60.382 & 12.44 \\
\hline \multirow{4}{*}{\multicolumn{2}{|c|}{ MNS (A2) }} & $\alpha_{c}=0.05$ & 45.424 & -0.301 & 59.350 & 10.52 \\
\hline & & $\alpha_{c}=0.10$ & 45.326 & -0.516 & 59.730 & 11.23 \\
\hline & & $\alpha_{c}=0.15$ & 45.377 & -0.404 & 58.932 & 9.739 \\
\hline & & $\alpha_{c}=0.20$ & 44.987 & -1.260 & 60.537 & 12.73 \\
\hline
\end{tabular}

Finally, a detailed investigation of the overall computational performances exhibited by the present multiscale algorithm and the considered alternative ones (i.e. algorithms A1 and A2) has been carried out; 
such performances have been measured by monitoring the cumulative number of total degrees of freedom (DOFs) over the incremental steps of the simulation, for the multiscale numerical simulations (MNSs) performed by using the present algorithm and those proposed in [50] (referred to as algorithms A1 and A2), and the direct numerical simulation (DNS), as shown in Table 6. Obviously, the number of total DOFs remains constant in the direct numerical simulation, for which a fine-scale model is employed everywhere from the beginning of analysis, and thus its cumulative value varies linearly with the incremental steps; on the other hand, the number of total DOFs varies during a single multiscale numerical simulation, as the zone of interest, i.e. the fully meshed subdomain, is progressively enlarged.

Table 6. Number of cumulative DOFs for the multiscale numerical simulations (MNSs) performed by using the present algorithm and those proposed in [50] (referred to as algorithms A1 and A2), computed at three different incremental steps.

\begin{tabular}{|c|c|c|c|c|c|}
\hline \multirow{2}{*}{\multicolumn{3}{|c|}{ DNS (reference) }} & $\delta=0.25 \mathrm{~mm}$ & $\delta=2.5 \mathrm{~mm}$ & $\delta=4.5 \mathrm{~mm}$ \\
\hline & & & 512,950 & $5,129,500$ & $8,207,200$ \\
\hline \multirow{16}{*}{ MNS (present) } & \multirow{4}{*}{$c=1 / 1.5$} & $\alpha_{c}=0.05$ & 45,680 & $3,947,312$ & $6,952,010$ \\
\hline & & $\alpha_{c}=0.10$ & 36,584 & $3,711,296$ & $6,580,136$ \\
\hline & & $\alpha_{c}=0.15$ & 36,584 & $3,760,870$ & $6,715,750$ \\
\hline & & $\alpha_{c}=0.20$ & 36,584 & $3,648,308$ & $6,572,020$ \\
\hline & \multirow{4}{*}{$c=1 / 2$} & $\alpha_{c}=0.05$ & 55,408 & $4,043,806$ & $7,125,964$ \\
\hline & & $\alpha_{c}=0.10$ & 43,230 & $3,945,068$ & $7,012,064$ \\
\hline & & $\alpha_{c}=0.15$ & 43,230 & $3,693,436$ & $6,725,776$ \\
\hline & & $\alpha_{c}=0.20$ & 43,230 & $3,680,392$ & $6,629,452$ \\
\hline & \multirow{4}{*}{$c=1 / 3$} & $\alpha_{c}=0.05$ & 75,282 & $4,188,428$ & $7,376,712$ \\
\hline & & $\alpha_{c}=0.10$ & 57,006 & $4,054,582$ & $7,198,360$ \\
\hline & & $\alpha_{c}=0.15$ & 57,006 & $3,770,586$ & $6,886,206$ \\
\hline & & $\alpha_{c}=0.20$ & 54,504 & $3,737,712$ & $6,770,052$ \\
\hline & \multirow{4}{*}{$c=1 / 5$} & $\alpha_{c}=0.05$ & 117,618 & $4,310,170$ & $7,472,666$ \\
\hline & & $\alpha_{c}=0.10$ & 85,996 & $4,148,916$ & $7,328,646$ \\
\hline & & $\alpha_{c}=0.15$ & 73,350 & $3,993,688$ & $7,100,980$ \\
\hline & & $\alpha_{c}=0.20$ & 65,068 & $3,799,680$ & $6,832,020$ \\
\hline \multicolumn{3}{|l|}{ MNS (A1) } & 29,396 & $3,604,064$ & $6,407,864$ \\
\hline \multirow{4}{*}{\multicolumn{2}{|c|}{ MNS (A2) }} & $\alpha_{c}=0.05$ & 208,476 & $4,555,344$ & $7,777,484$ \\
\hline & & $\alpha_{c}=0.10$ & 122,084 & $4,237,942$ & $7,417,672$ \\
\hline & & $\alpha_{c}=0.15$ & 103,656 & $3,918,462$ & $7,034,082$ \\
\hline & & $\alpha_{c}=0.20$ & 82,286 & $3,826,404$ & $6,858,744$ \\
\hline
\end{tabular}

All the considered algorithms, i.e. the present algorithm and those introduced in [50] and there referred to as A1 and A2, lead to comparable computational costs; moreover, the following considerations can be drawn:

1) The computational cost of the present multiscale algorithm is globally reduced by increasing the parameter $\alpha_{c}$ at fixed $c$, or by increasing the parameter $c$ at fixed $\alpha_{c}$.

2) The present multiscale algorithm exhibits the best computational performances with $\alpha_{c}=0.20$ and $c=$ $1 / 1.5$, for which the sum of total number of DOFs for all the incremental steps reaches its minimum value among all the considered cases.

3) Algorithms A2 is less efficient than algorithm A1, but its performances tend to achieve the same level as algorithm A1 for increasing values of $\alpha_{c}$.

4) The worst performances are found by using algorithm A2 with $\alpha_{c}=0.05$, for which the sum of total number of DOFs for all incremental steps reaches its maximum value among all the considered cases. 
5) The computational performances of the present multiscale algorithm tend to those of algorithm A1 for higher values of $c$ (i.e. for $c$ going to 1 ) and to those of algorithm A2 for smaller values of $c$ (i.e. for $c$ going to 0$)$.

Finally, by intersecting the results obtained by the three multiscale algorithms in terms of both accuracy and computational efficiency, one may observe that the newly proposed algorithm represents an optimal compromise between the needs of reducing the computational costs and of finding numerical results as accurate as possible. For the considered case, the optimal parameter values are $\alpha_{c}=0.20$ and $c=1 / 1.5$, leading to a very small error on the peak load with respect to the direct numerical simulation (of about $0.4 \%$ ) and a DOF ratio (computed as the summation of total DOF for all incremental steps) of the multiscale to the direct analysis of about $80 \%$; such a result correspond to a limited speed-up, essentially due to the fact that, under the considered loads and boundary conditions, the masonry panel is subjected to an extensive damage until the peak load is reached, thus triggering the zooming-in operations in a very large portion of the computational domain, as already observed in Fig. 13. Conversely, for the same case, the best computational performances are experienced at the early damage states (i.e. for $\delta=0.25 \mathrm{~mm}$ ), for which a cumulative DOF ratio between MNS and DNS of about 7\% is obtained, corresponding to a high speed-up value.

\section{Application to masonry with fiber-reinforced mortar joints: the deep beam test}

The second application is devoted to the investigation of the mechanical behavior up to failure of masonry structures with fiber-reinforced mortar joints under general loading conditions; such an application consists in the deep beam test introduced in [61] for masonry with traditional mortar joints, and here proposed for masonry with fiber-reinforced mortar joints. Such a test, which is characterized by a combined shear-flexure structural response as the test reported in Section 4, has been extensively employed for calibrating a number of numerical models of masonry (see [62,30]).

\subsection{Material and geometric properties}

The considered test specimen, whose geometry and boundary conditions are shown in Fig. 16, is a smallsized deep beam with dimensions $757 \times 457 \mathrm{~mm}^{2}$, resulting in a width/height ratio of about 1.66; such a specimen is rigidly supported at each end over a length of $188 \mathrm{~mm}$, meaning that no rotations and relative displacements are allowed at the supports. The top load $P$, which is supposed to be applied via a stiff steel beam, is simulated by a prescribed vertical displacement $\delta$ uniformly distributed over a length of $381 \mathrm{~mm}$ and monotonically increasing up to failure.

This beam is made of half-scale solid clay bricks of $122 \times 37 \times 54 \mathrm{~mm}^{3}$ in dimension, separated by mortar joints of $5 \mathrm{~mm}$ in thickness. The elastic behavior of brick material is assumed to be linearly isotropic, and characterized by the material constants $E_{b}=7,400 \mathrm{MPa}$ and $v_{b}=0.167$, whereas the elastic behavior of mortar joints, which are initially undamaged, is defined by means of the normal and tangential stiffness components, i.e. $K_{n}$ and $K_{s}$, obtained via Eq. (4), which in turn depend on the material constants of both mortar and bricks; in this section, three mortar types have been compared, i.e. a traditional (M0) and two basalt fiber-reinforced (M1 and M2) mortars with different fiber contents (expressed as a percentage with respect to the solid fraction of the mixture). The adopted elastic constants of all the mortar types are $E_{m}=$ $788 \mathrm{MPa}$ and $v_{m}=0.167$; as a matter of fact, the presence of randomly distributed short fibers does not affect the elastic properties of mortar joints, owing to the low fiber content (which generally amounts to less than $5 \%)$.

For this test, unlike the previous example, bricks are damageable, and thus the sources of nonlinearity rely on the inelastic behavior of both units and mortar joints, according the cohesive traction-separation law discussed in Section 2.1. The required inelastic parameters of mortar are the uniaxial tensile, uniaxial compressive and biaxial compressive strengths, denoted by $\sigma_{t}, \sigma_{c}$ and $\sigma_{b}$, respectively, as well as the mode-I 
fracture energy $G_{f}$; as usual, the latter quantity is obtained from the fracture energy per unit volume of mortar, denoted as $G_{f, 1}$, accepting that damage is allowed to spread on the entire joint thickness (see [57]).

The most important effect of the fiber reinforcement is the significant increase in the fracture toughness of the mortar for increasing values of fiber volume ratio; however, for a given water content, there exists an optimum dosage of fiber reinforcement, beyond which the reinforcement efficiency does not increase; such a behavior is believed to be attributed to the experienced difficulty in mix compaction and fiber dispersion (see [13]). The fiber-reinforced mortars considered for this example are made of randomly distributed short basalt fibers with mean length of about $5 \mathrm{~mm}$; their properties in terms of fiber content and fracture energy per unit volume, listed in Table 7, have been found in the literature (see [12]). It is worth noting that the strength parameters are scarcely influenced by the presence of such a reinforcement (see, for instance, [12]); as a consequence, the strength properties of mortar joints adopted in the following computations are taken as constants, assuming $\sigma_{t}=0.29 \mathrm{~N} / \mathrm{mm}^{2}, \sigma_{c}=8.6 \mathrm{~N} / \mathrm{mm}^{2}$, and $\sigma_{b}=10.3 \mathrm{~N} / \mathrm{mm}^{2}$.

Furthermore, unlike the previously discussed shear wall test, in this test bricks are susceptible to be damaged, and thus mode-I cohesive elements are placed along predefined weak planes inside bricks, according to the mesoscopic modeling discussed in Section 2.1; the required parameters, i.e. the tangential and normal penalty stiffness parameters $K_{s}$ and $K_{n}$, the tensile strength $\sigma_{t}$ and the mode-I fracture energy $G_{f}$, are listed in Table 8.

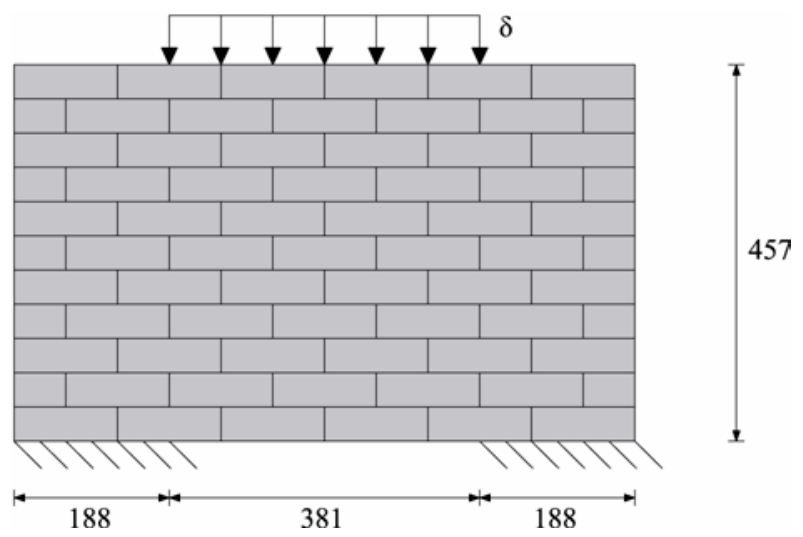

Fig. 16. Deep beam test for a masonry with fiber-reinforced mortar joints: geometry and boundary conditions (all dimensions are expressed in millimeters).

Table 7. Fiber content, fracture energy per unit volume and fracture energy of traditional and fiberreinforced mortar (fiber content is expressed as a percentage with respect to the solid fraction of the mixture).

\begin{tabular}{|c|c|c|c|}
\hline Mortar type & Fiber content [\%] & $G_{f, 1}\left[\mathrm{~N} / \mathrm{mm}^{2}\right]$ & $G_{f}[\mathrm{~N} / \mathrm{mm}]$ \\
\hline M0 & 0 & 0.01 & 0.05 \\
\hline M1 & 1 & 0.04 & 0.20 \\
\hline M2 & 2 & 0.05 & 0.25 \\
\hline
\end{tabular}

Table 8. Properties for the potential cracks inside bricks.

\begin{tabular}{|c|c|c|c|}
\hline$K_{s}\left[\mathrm{~N} / \mathrm{mm}^{3}\right]$ & $K_{n}\left[\mathrm{~N} / \mathrm{mm}^{3}\right]$ & $\sigma_{t}\left[\mathrm{~N} / \mathrm{mm}^{2}\right]$ & $G_{f}[\mathrm{~N} / \mathrm{mm}]$ \\
\hline $1.0 \mathrm{e} 6$ & $1.0 \mathrm{e} 6$ & 2.0 & 0.10 \\
\hline
\end{tabular}

\subsection{Multiscale numerical simulation (MNS) results}


As for the previous example, the first step to be performed within the proposed multiscale strategy is the determination of both the (undamaged) homogenized moduli and the first failure surface for the given masonry, according to the procedures presented in Sections 2.2 and 2.4, respectively. The relevant numerical computations have been carried out on the same repeating cell as in Section 4.2, assuming a plane stress state and periodic boundary conditions, and adopting the cross-triangle quadrilateral mesh depicted in Fig. 17; such a mesh has been chosen to accommodate the placement of cohesive interface elements inside the bricks, along the inclined orientations, as shown in Fig. 3, needed for the subsequent nonlinear analyses.

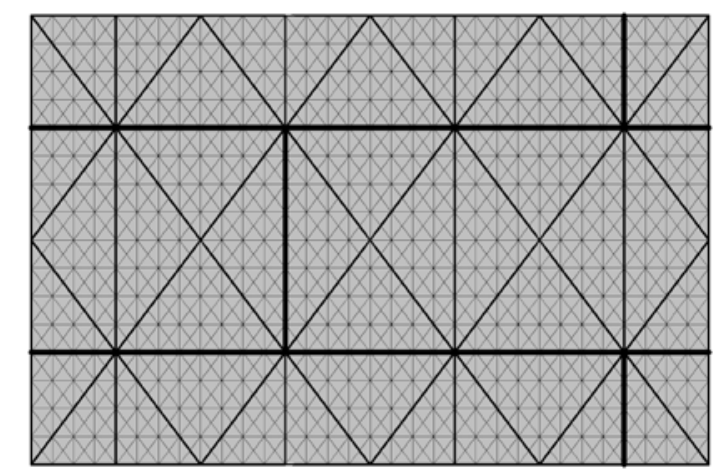

Fig. 17. Adopted mesh for the considered RC of the deep beam.

The predicted overall moduli of undamaged masonry are shown in Table 9, whereas the numerically derived first failure locus is sketched in Fig. 18, as a one-parameter family of contour lines in the principal macrostrain plane.

Table 9. Homogenized moduli for the in-plane response of the given masonry microstructure.

\begin{tabular}{|r|r|r|r|r|r|}
\hline$C_{1111}^{\text {hom }}[\mathrm{GPa}]$ & $C_{1122}^{\text {hom }}[\mathrm{GPa}]$ & $C_{1112}^{\text {hom }}[\mathrm{GPa}]$ & $C_{2222}^{\text {hom }}[\mathrm{GPa}]$ & $C_{2212}^{\text {hom }}[\mathrm{GPa}]$ & $C_{1212}^{\text {hom }}[\mathrm{GPa}]$ \\
\hline 5.855 & 0.489 & 0 & 3.743 & 0 & 1.425 \\
\hline
\end{tabular}

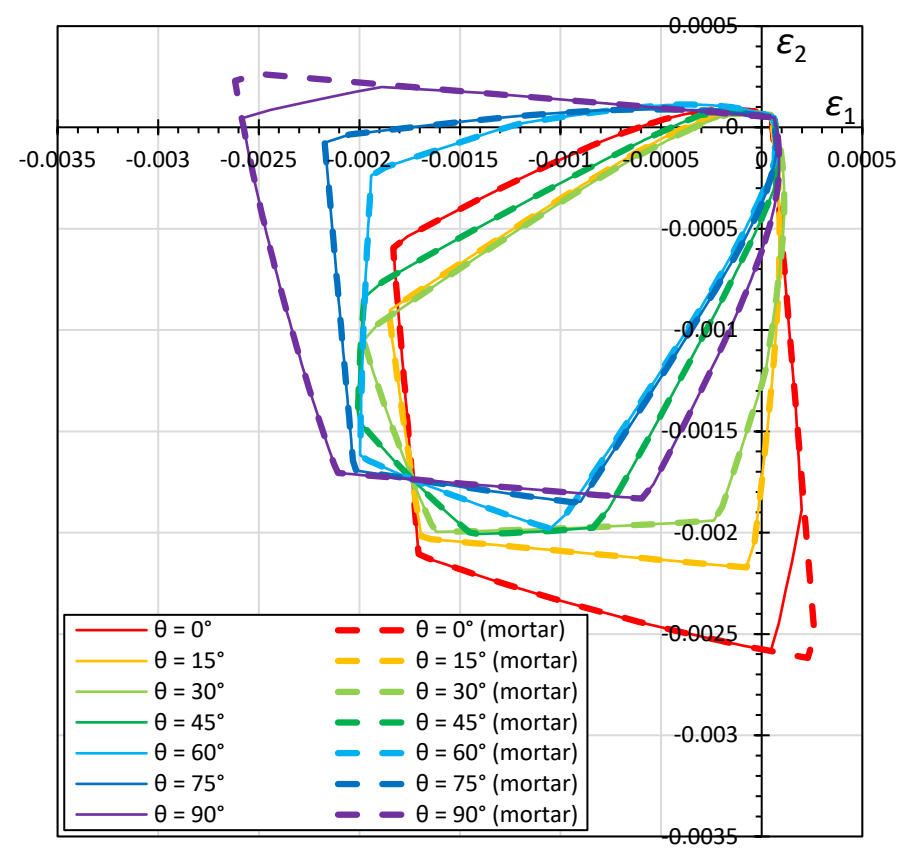


Fig. 18. Microscopically informed first failure locus for periodic masonry with fiber-reinforced mortar joints, represented as a one-parameter family of contour lines in the principal macrostrain plane.

Unlike the first failure locus shown in Fig. 11, the present one (see continuous lines of Fig. 18) takes into account not only cracking of mortar joints, but also damage mechanisms inside the bricks, coherently with the assumed relatively low toughness of units with respect to fiber-reinforced mortar joints; however, this locus does not depend on the fracture energy of micro-constituents, as it concerns the crack initiation phenomenon rather than the subsequent crack propagation, and therefore a unique first failure surface is assumed to be valid for both traditional and fiber-reinforced mortar joints. As a consequence, the inclusion of possible damage mechanisms inside the bricks is expected not to affect the size of first failure domain; in order to verify this, another locus has been constructed by considering only damage initiation at mortar joints (see dashed lines of Fig. 18). Surprisingly, these two loci do not coincide completely; in fact, for a few tested macrostrain directions, the first failure locus obtained considering crack initiation within the units is found to be more restrictive than the other one; it means that crack initiation at mortar joints precedes that inside the bricks, except for a few macrostrain directions. Interestingly, it can be observed that these discrepancies occur when the principal macrostrain directions are aligned with the orthotropic axes.

After this, different multiscale numerical simulations have been carried out, using the proposed adaptive multiscale strategy and assuming $\alpha_{c}=0.10$ and $c=1 / 3$; these values have been chosen as intermediate values in the ranges considered in the previous sensitivity analysis. The resulting structural responses numerically predicted for the three considered mortar types have been superposed in Fig. 19. It can be observed that the use of a fiber-reinforced mortar leads to a considerable increase in the ductility of the masonry beam; the ultimate displacements numerically obtained for the cases M1 and M2 are greater than 35\% of the ultimate displacement registered for the traditional mortar. It is believed that the actual value of the ductility ratio is even greater than the one reported here, due to the employed nonlinear solver (based on Newton's iterations); a lack of convergence has been experienced for all the numerical computations, probably due to the appearance of local snap-back phenomena.

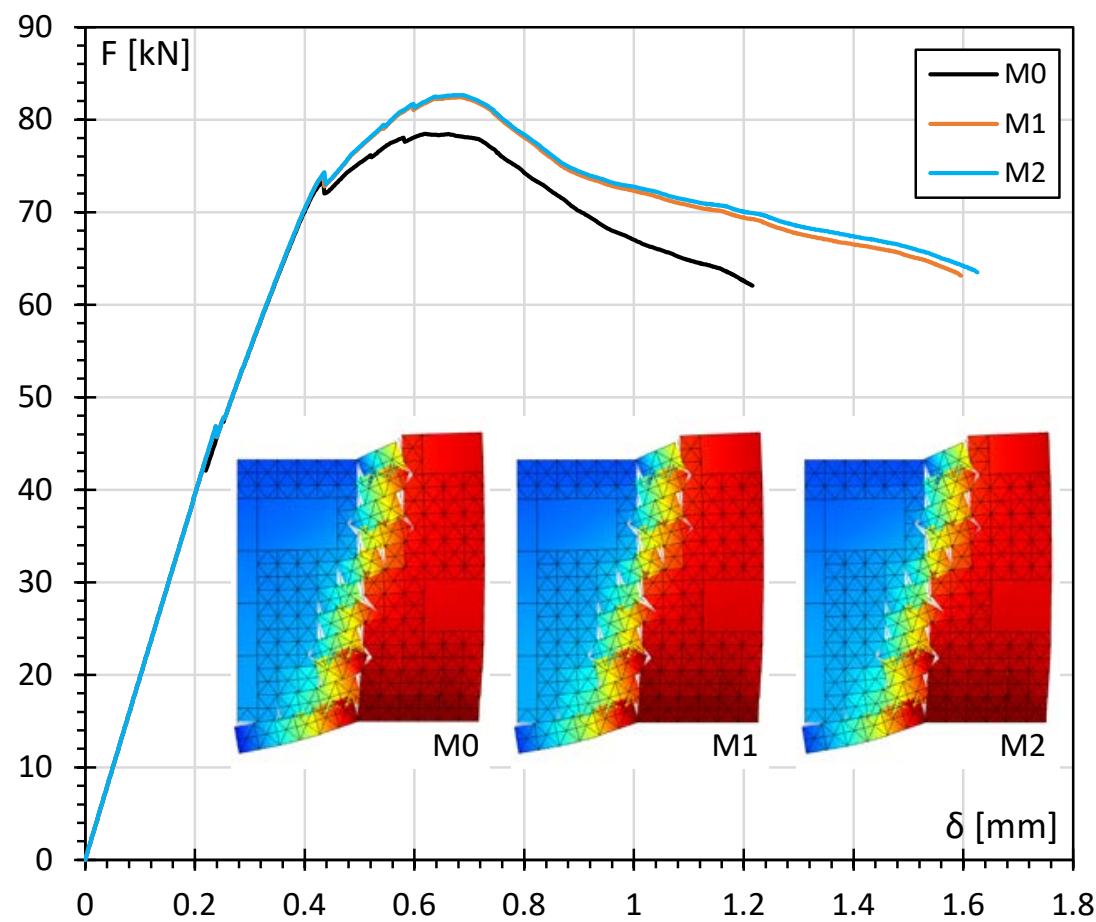

Fig. 19. Force versus displacement curve obtained from the multiscale numerical simulation (MNS) performed according to the proposed algorithm (with $\alpha_{c}=0.10$ and $c=1 / 3$ ), for the deep masonry beam with traditional (case M0) and reinforced mortar (cases M1 and M2). 
At the same time, the use of a fiber-reinforced mortar leads to a little increase in the peak load, which is of about 5\% for both cases M1 and M2. Interestingly, the structural responses associated with the use of fiberreinforced mortar joints with different fiber content are quite similar to each other; it means that using a mortar with a fiber content up to $1 \%$ provides a significant toughening effect, but a further increase in the fiber content does not contribute to any improvement in this direction.

The damage patterns at the ultimate stages of simulation associated with the three considered mortar types are represented in Fig. 20; all these pattern, determined for $\delta=1.2 \mathrm{~mm}$, are very similar to each other, but in the presence of fiber-reinforced mortar joints, i.e. in the cases (b) and (c), the number of cracked bricks is greater than in the case (a). This is due to the fact the toughening effect provided by the fiber-reinforced mortar ultimately leads to a major number of crack arrests along the joints and of crack initiation inside the units.

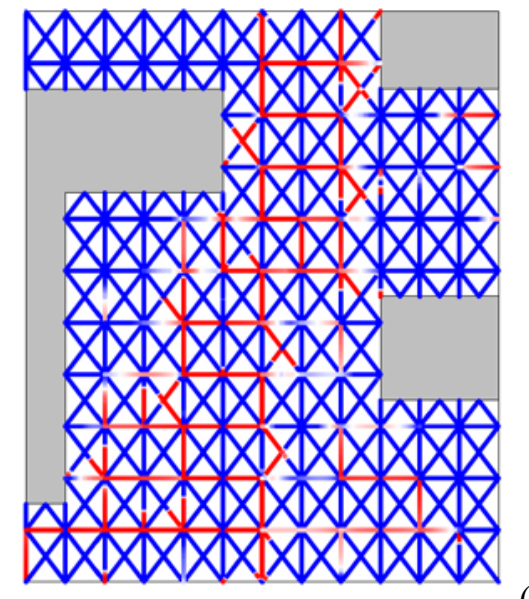

(a)

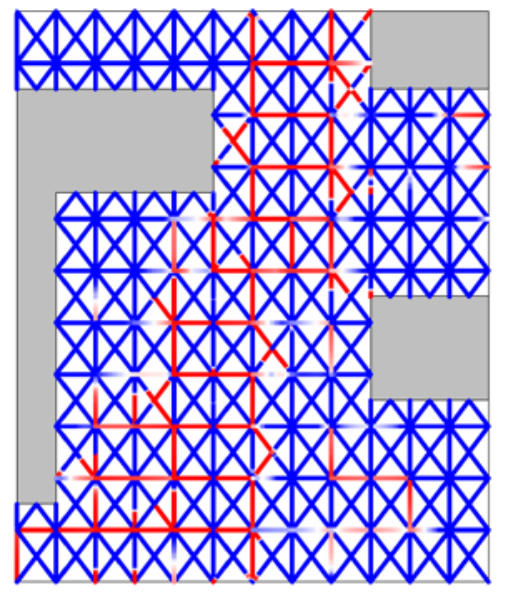

(b)

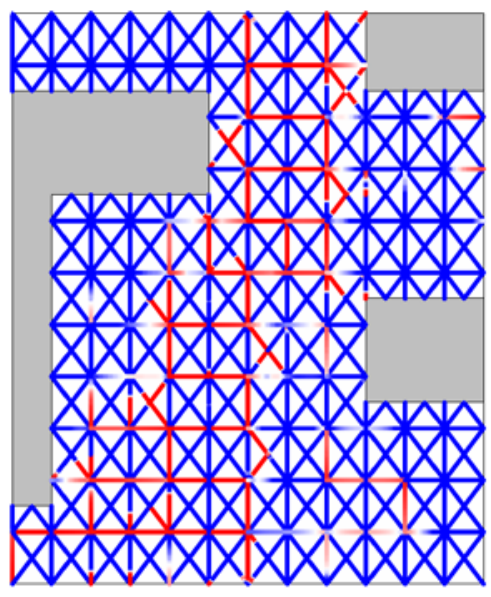

(c)

Fig. 20. Damage variable maps for $\delta=1.2 \mathrm{~mm}$ obtained from the multiscale numerical simulation (MNS) performed according to the proposed algorithm (with $\alpha_{c}=0.10$ and $c=1 / 3$ ), for the deep masonry beam with traditional (case M0) and reinforced mortar (cases M1 and M2); the coarse-level elements outside the zone of interest are shaded in grey, whereas the damage variable defined on the cohesive interfaces ranges from 0 (blue) to 1 (red); (a) case M0; (b) case M1; (c) case M2.

As expected, a limited number of discontinuity points is identified, which potentially affects the accuracy of the obtained numerical results; however the maximum value attained by the a posteriori error defined by Eq. (7) is of about 3\% for all the considered mortar types, as shown in Table 10; this confirms the validity of the proposed multiscale strategy for the nonlinear analysis of masonry structures with both traditional (M0) and fiber-reinforced (M1 and M2) mortar joints.

Table 10. Estimation of the a posteriori error $e_{F}$ (expressed as a percent) for assessing the validity of the present multiscale strategy applied to a masonry deep beam with both traditional (M0) and fiber-reinforced (M1 and M2) mortar joints.

\begin{tabular}{|c|c|}
\hline Mortar type & $e_{F}[\%]$ \\
\hline M0 & 3.082 \\
\hline M1 & 3.341 \\
\hline M2 & 3.341 \\
\hline
\end{tabular}

\subsection{Validation with direct numerical simulation (DNS) results}


The predicted structural response of the deep masonry beam with traditional and fiber-reinforced mortar joints, obtained by using the proposed adaptive multiscale strategy, has been validated by comparing it with that obtained by a direct numerical simulation, performed on a fully meshed specimen.

Fig. 15 shows a comparison in terms of force versus displacement curve between the multiscale (MNS) and the direct numerical simulation (DNS), for the deep masonry beam with both traditional and fiber-reinforced mortar joints; it can be observed that the proposed multiscale strategy leads to very accurate numerical results for both the peak and the post-peak responses, for the three considered mortar types.

In order to better highlight the accuracy level of the multiscale numerical simulations, the peak load and the initial stiffness predicted by the multiscale numerical simulations are shown in Table 11, together with the percentage errors with respect to the corresponding direct numerical simulations; the errors on the peak load have been found to be very small (less than 1\%) for all the considered mortar types; in particular, the smallest errors are associated with the cases M1 and M2, which are of about $0.5 \%$. On the other hand, the errors on the initial stiffness are practically the same for the different mortar types, remaining systematically overestimated of about $5 \%$. These results confirm the validity of the proposed multiscale strategy in terms of numerical accuracy, for the damage analysis of masonry structures with both traditional and fiber-reinforced mortar joints.
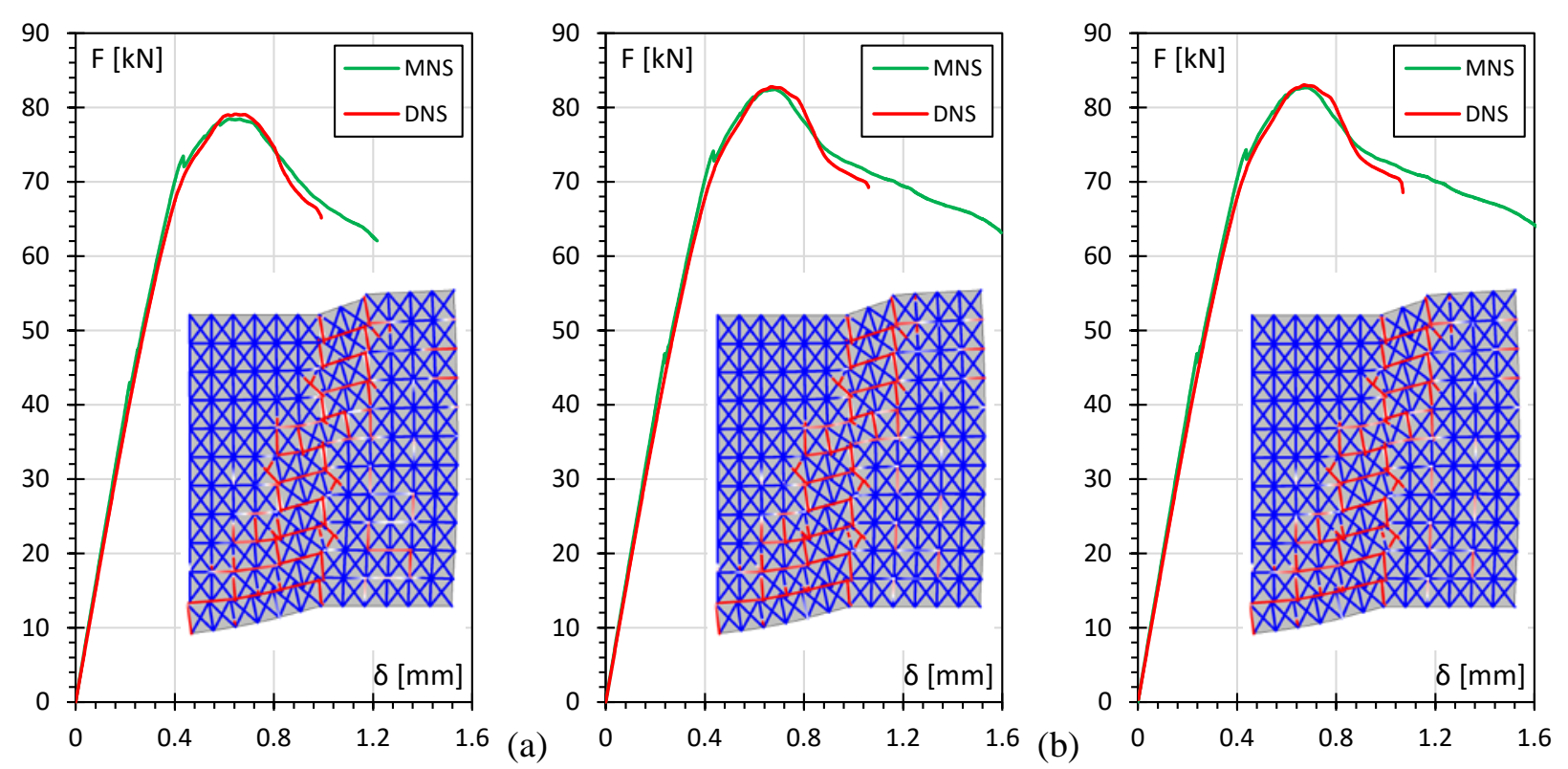

Fig. 21. Comparison in terms of force versus displacement curve between the multiscale (MNS) and the direct (DNS) numerical simulation (DNS) for the deep masonry beam with traditional (case M0) and reinforced mortar (cases M1 and M2); (a) case M0; (b) case M1; (c) case M2.

Table 11. Comparisons in terms of predicted peak load and initial stiffness between the multiscale numerical simulations (MNSs) performed by using the present algorithm and the direct numerical simulation (DNS).

\begin{tabular}{|l|l|r|r|r|r|}
\hline \multicolumn{2}{|c|}{} & \multicolumn{2}{|c|}{ Peak load } & \multicolumn{2}{l|}{ Initial stiffness } \\
\cline { 3 - 6 } \multicolumn{2}{|c|}{} & $F_{\max }[\mathrm{kN}]$ & Error [\%] & $K_{0}[\mathrm{kN} / \mathrm{mm}]$ & Error [\%] \\
\hline \multirow{2}{*}{ M0 } & DNS (reference) & 79.095 & & 188.6 & \\
\cline { 2 - 6 } & MNS & 78.435 & -0.8344 & 198.3 & 5.143 \\
\hline \multirow{2}{*}{ M1 } & DNS (reference) & 82.788 & & 188.6 & \\
\cline { 2 - 6 } & MNS & 82.399 & -0.4699 & 198.3 & 5.143 \\
\hline \multirow{2}{*}{ M2 } & DNS (reference) & 83.033 & & 188.6 & \\
\cline { 2 - 6 } & MNS & 82.647 & -0.4649 & 198.3 & 5.143 \\
\hline
\end{tabular}


Finally, the computational performances exhibited by the present multiscale method have been investigated, by monitoring the cumulative number of total degrees of freedom (DOFs) over the incremental steps of the simulation, computed at three different incremental steps, for both the multiscale (MNS) and the direct (DNS) numerical simulations, as shown in Table 12.

The resulting cumulative DOF ratio (computed as the summation of total DOF for all incremental steps) of the multiscale to the direct analysis is almost independent on the considered mortar type, since the extension of the damaged region predicted by the multiscale numerical simulation is essentially the same for M0, M1 and M2 types, with limited discrepancies only in the local damage pattern, as already observed in Fig. 20. The average DOF ratios for the incremental steps $\delta=(0.10 ; 0.60 ; 1.0) \mathrm{mm}$ are of about $20 \%, 50 \%$ and $60 \%$. Such results correspond to a higher speed-up with respect to the shear wall test; this is essentially due to the fact that the deep masonry beam is subjected to a less spread damage distribution, being localized within a restricted band, thus triggering the zooming-in operations in a small portion of the computational domain.

Table 12. Number of cumulative DOFs for the multiscale numerical simulations (MNSs) performed by using the present algorithm, computed at three different incremental steps.

\begin{tabular}{|l|l|r|r|r|}
\hline \multicolumn{2}{|c|}{} & $\delta=0.10 \mathrm{~mm}$ & $\delta=0.60 \mathrm{~mm}$ & $\delta=1.0 \mathrm{~mm}$ \\
\hline \multirow{2}{*}{ M0 } & DNS (reference) & $3,000,000$ & $18,000,000$ & $30,000,000$ \\
\cline { 2 - 5 } & MNS & 572,488 & $9,197,700$ & $18,806,100$ \\
\hline \multirow{2}{*}{ M1 } & DNS (reference) & $3,000,000$ & $18,000,000$ & $30,000,000$ \\
\cline { 2 - 5 } & MNS & 572,488 & $9,108,044$ & $18,716,444$ \\
\hline \multirow{2}{*}{ M2 } & DNS (reference) & $3,000,000$ & $18,000,000$ & $30,000,000$ \\
\cline { 2 - 5 } & MNS & 572,488 & $9,098,934$ & $18,707,334$ \\
\hline
\end{tabular}

\section{Concluding remarks}

A refined multiscale model adopting a two-level domain decomposition technique and an adaptive refinement strategy has been proposed here to predict the nonlinear behavior of periodic masonry structural elements subjected to general in-plane loading conditions. The complex damage evolution process is analyzed by taking into account crack initiation and propagation in both masonry blocks and mortar joints. Zone of incipient damage localization for which a fine level description is needed are detected by means of mesoscopically informed first failure surfaces taking into account higher-order deformation effects.

Such a strategy, which falls into the wide class of concurrent multiscale methods, is able to overcome the limitations of classical (continuous) first-order homogenization methods, which inevitably fail due to the illposedness of the resulting macroscopic problem in the presence of localization. Furthermore, the proposed method is believed by the authors to perform better than alternative existing methods, mainly based on a semi-concurrent approach; indeed, its main advantage relies in the possibility of handling both boundary layer effects (free edges, concentrated loads, etc.) and damage nucleation phenomena, since scale separation assumption is only imposed up to the microcrack nucleation but not during the subsequent microcrack propagation.

Model adaptivity is the fundamental concept underlying the proposed multiscale approach, since it plays a central role in affecting at the same time the overall computational burden of the method and the accuracy of the related numerical results. As a matter of fact, the main novelty of the adopted strategy is a refined zooming-in criterion to be applied within the above-mentioned adaptive model refinement strategy; such a criterion represents a generalized version of the two criteria proposed in the authors' previous work [50], which is here found to be more flexible in obtaining an optimal balance between accuracy and computational efficiency. Such a balance could be reached by performing a fine tuning of the two numerical parameters of the model, i.e. the threshold hourglass energy ratio $\alpha_{c}$ and scale factor of the first failure locus $c$. 
Another aspect of novelty is a more accurate damage representation for periodic masonry structures than the existing ones incorporating, besides cohesive interface elements placed along the mortar joints, often used in the literature, also cohesive interface elements placed along both vertical and inclined lines inside the masonry units. Such a damage representation is able to accurately capture all the fracture mechanisms of masonry, including the diagonal tensile cracking of bricks which appears when masonry is subjected to mixed-mode loading conditions. All the cohesive interface elements within units and along joints have been equipped with phenomenological constitutive laws introduced in existing studies and appropriately adapted to the developed analyses.

Applications of the proposed multiscale masonry model have been carried out firstly with reference to a shear wall test in order to check its validity as the required main input parameters change and to calibrate the model internal parameters. This preliminary analysis has allowed to assess the validity and the efficiency of the proposed model through comparisons with direct fine-scale simulations and to tune its parameters in order to achieve an optimum balance between computational efficiency and numerical accuracy. Then a series of numerical experiments have been carried out for an innovative masonry deep beam application with fiber-reinforced mortar joints able to investigate the overall toughening effect associated with different fiber fractions.

Through these numerical investigations the following conclusions have been reached.

- The first failure loci of the given masonry with fiber-reinforced mortar joints determined in the principal macrostrain plane have showm that the mesoscopic crack initiation along the mortar joints generally precede crack nucleation inside the bricks, except for a few macrostrain path directions, limited to the case of principal macrostrain directions aligned with the orthotropic axes.

- The subsequent multiscale numerical simulations performed on the deep masonry beam have pointed out that the use of a mortar reinforced with randomly distributed short fibers with low volume fractions leads to a significant toughening, resulting in a considerable increase in the overall ductility at the structural level. In particular, an increase in the peak load of about $5 \%$ and an increase in the ultimate displacement of more than 35\% have been registered for the tested fiber-reinforced mortars (with fiber contents of $1 \%$ and $2 \%$ ); it is worth noting that very similar mechanical responses have been obtained by varying the fiber contents, meaning that using a fiber content of more than $1 \%$ does not contribute to any improvement in the overall toughness of considered masonry; obviously, such a threshold value has not a general validity, as it depends on the specific brick to mortar relative fracture energy.

- The results obtained by the multiscale numerical simulations have been validated by suitable comparisons with those coming from the corresponding direct numerical simulations, for which a fully meshed model has been employed. The proposed multiscale strategy has been found to predict in a very accurate manner both the peak and the post-peak responses, for the three considered mortar types; in particular, the errors on the peak load have been found to be always very small (less than $1 \%)$. The high level of accuracy obtained in the numerical experiments is mainly due to the fact that the proposed multiscale strategy does not introduce any simplification in the structural representation beyond the elastic limit of the masonry material, thus allowing an accurate evaluation of the damage development under general loading conditions.

- As far as the computational performances exhibited by the present multiscale method is concerned, the cumulated number of total DOFs during the multiscale numerical simulations has been monitored, for the three considered mortar types. A rather good speed-up value has been found, associated with a maximum value of the multiscale to direct DOF ratio of about $60 \%$; such a value is specific for the considered structure, material and boundary conditions, and is susceptible to be reduced for even more localized damage situations.

Inspired by the present numerical results, the following developments and improvements of the present multiscale method could be object of future investigations. Since in the proposed multiscale approach only zooming-in operations are performed, the model refinement turns out to be irreversible, meaning that already 
zoomed-in regions are no longer concerned with the homogenization process; future research could focus on the development of a refined approach, equipped with an additional zooming-out strategy to homogenize again the already damaged regions whose stiffness does not evolve anymore due to damage saturation. In addition a more sophisticated multiscale model could be developed, which is able to capture the influence of higher-order deformation modes on both linear and nonlinear ranges of the structural response of periodic masonry subjected to arbitrary loading conditions; such model could use improved homogenized moduli and first failure loci, to be obtained by explicitly introducing an enriched kinematics at the macroscopic scale, for instance by using the Cosserat continuum model. In such a case the incorporation of the micropolar deformation modes could improve significantly the accuracy in the determination of the load-carrying capacity of masonry structures, also capturing the size effects usually experienced in both linear and nonlinear ranges.

\section{References}

[1] Triantafillou, T.C. Strengthening of masonry structures using epoxy-bonded FRP laminates (1998) Journal of Composites for Construction, 2 (2), pp. 96-104.

[2] R. Luciano, E. Sacco, Damage of masonry panels reinforced by FRP sheets, International Journal of Solids and Structures, Volume 35, Issue 15, 1998, Pages 1723-1741.

[3] Ascione L., Feo L., Fraternali F. (2005), Load carrying capacity of 2D FRP/strengthened masonry structures. Composites Part B: Engineering, 36(8): 619-626.

[3] Ghiassi B., Oliveira D.V., Lourenço P.B., Marcari G. (2013), Numerical study of the role of mortar joints in the bond behavior of FRP-strengthened masonry. Composites Part B: Engineering, 46: 21-30.

[4] Caporale A, Feo L, Hui D, Luciano R. Debonding of FRP in multi-span masonry arch structures via limit analysis. Compos Struct 2014;108:856-65.

[5] Marcari G., Manfredi G., Prota A., Pecce M. (2007), In-plane shear performance of masonry panels strengthened with FRP. Composites Part B: Engineering, 38: 887-901.

[6] A. Caporale, L. Feo, R. Luciano, R. Penna, Numerical collapse load of multi-span masonry arch structures with FRP reinforcement, Composites Part B: Engineering, Volume 54, November 2013, Pages 7184.

[7] D’Ambrisi A., Feo L., Focacci F. (2013), Experimental and analytical investigation on bond between Carbon-FRCM materials and masonry. Composites Part B: Engineering, 46: 15-20.

[8] Carozzi F.G., Poggi C. (2015), Mechanical properties and debonding strength of Fabric Reinforced Cementitious Matrix (FRCM) systems for masonry strengthening. Composites Part B: Engineering, 70: 215230.

[9] Garofano A., Ceroni F., Pecce M. (2016), Modelling of the in-plane behaviour of masonry walls strengthened with polymeric grids embedded in cementitious mortar layers. Composites Part B: Engineering, 85: 243-258.

[10] Erdogmus E. (2015), Use of Fiber-Reinforced Cements in Masonry Construction and Structural Rehabilitation. Fibers, 3(1): 41-63.

[11] Asprone D., Cadoni E., Iucolano F., Prota A. (2014), Analysis of the strain-rate behavior of a basalt fiber reinforced natural hydraulic mortar. Cement \& Concrete Composites, 53: 52-58.

[12] Iucolano F., Liguori B., Colella C., Fibre-reinforced lime-based mortars: A possible resource for ancient masonry restoration. Construction and Building Materials 38 (2013) 785-789. 
[13] Chan R., Bindiganavile V. (2010), Toughness of fibre reinforced hydraulic lime mortar. Part-1: Quasistatic response. Materials and Structures, 43(10): 1435-1444.

[14] Chan R., Bindiganavile V. (2010), Toughness of fibre reinforced hydraulic lime mortar. Part-2: Dynamic response. Materials and Structures, 43(10): 1445-1455.

[15] Maria Laura Santarelli, Francesca Sbardella, Martina Zuena, Jacopo Tirillò, Fabrizio Sarasini, Basalt fiber reinforced natural hydraulic lime mortars: A potential bio-based material for restoration, Materials and Design 63 (2014) 398-406.

[16] Jean M. (1999), The non-smooth contact dynamics method. Comput. Meth. Appl. Mech. Eng., 177(3): 235-257.

[17] Chetouane, B., Dubois, F., Vinches, M., \& Bohatier, C. (2005). NSCD discrete element method for modelling masonry structures. International journal for numerical methods in engineering, 64(1), 65-94.

[18] Lourénço P.B., De Borst R. and Rots J.G. (1997), A plane stress softening plasticity model for orthotropic materials. Int. J. Numer. Meth. Engng., 40: 4033-4057.

[19] Berto L., Saetta A., Scotta R. and Vitaliani R. (2002), An orthotropic damage model for masonry structures. Int. J. Numer. Meth. Engng., 55: 127-157.

[20] Pelà L., Cervera M. and Roca P. (2011), Continuum damage model for orthotropic materials: Application to masonry. Comput. Meth. Appl. Mech. Eng., 200(9-12): 917-930.

[21] Anthoine A. (1995), Derivation of the in-plane elastic characteristics of masonry through homogenization theory. Int. J. Solids Struct., 32(2): 137-163.

[22] Luciano R., Sacco E. (1997), Homogenization technique and damage model for old masonry material. Int. J. Solids Struct., 34(24): 3191-3208.

[23] Greco F., Leonetti L., Nevone Blasi P. (2012), Non-linear macroscopic response of fiber-reinforced composite materials due to initiation and propagation of interface cracks. Eng. Fract. Mech., 80: 92-113.

[24] Caporale A., Parisi F., Asprone D., Luciano R., Prota A. (2014), Critical surfaces for adobe masonry: Micromechanical approach. Composites Part B: Engineering, 56: 790-796.

[25] Feyel F., Chaboche J.L. (2000), FE2 multiscale approach for modelling the elastoviscoplastic behaviour of long fibre SiC/Ti composite materials. Comput. Method. Appl. Mech., 183(3-4): 309-330.

[26] Kouznetsova V.G., Geers M.G.D., Brekelmans W.A.M. (2004), Multi-scale second-order computational homogenization of multi-phase materials: a nested finite element solution strategy. Comput. Method. Appl. Mech., 193(48-51): 5525-5550.

[27] Masiani R, Trovalusci P. Cauchy and Cosserat materials as continuum models of brick masonry. Meccanica 1996;31(4):421-32.

[28] Trovalusci P, Capecchi D, Ruta G. Genesis of the multiscale approach for materials with microstructure. Arch Appl Mech 2009;79:981-97.

[29] Addessi D, Sacco E, Paolone A. Cosserat model for periodic masonry deduced by nonlinear homogenization. Eur J Mech A/Solids 2010;29:724-37.

[30] De Bellis ML, Addessi D. A cosserat-based multi-scale model for masonry structures. Int J Multiscale Comput Eng 2011;9(5):543-63.

[31] Pau A, Trovalusci P. Block masonry as equivalent micropolar continua: the role of relative rotations. Acta Mech 2012;223(7):1455-71. 
[32] Trovalusci P, Pau A. Derivation of microstructured continua from lattice systems via principle of virtual works: the case of masonry-like materials as micropolar, second gradient and classical continua. Acta Mech 2014;225(1):157-77.

[33] Trovalusci P, Ostoja-Starzewski M, De Bellis ML, Murrali A. Scale-dependent homogenization of random composites as micropolar continua. Eur J Mech A/Solids 2015;49:396-407.

[34] Fabrizio Greco, A study of stability and bifurcation in micro-cracked periodic elastic composites including self-contact, International Journal of Solids and Structures, Volume 50, Issue 10, 15 May 2013, Pages 1646-1663.

[35] Domenico Bruno, Fabrizio Greco, Raimondo Luciano, Paolo Nevone Blasi, Nonlinear homogenized properties of defected composite materials, Computers \& Structures, Volume 134, 1 April 2014, Pages 102111.

[36] Fabrizio Greco, Lorenzo Leonetti, Raimondo Luciano, Paolo Nevone Blasi, Effects of microfracture and contact induced instabilities on the macroscopic response of finitely deformed elastic composites, Composites Part B: Engineering, Volume 107, 15 December 2016, Pages 233-253.

[37] Fabrizio Greco, Lorenzo Leonetti, Paolo Lonetti, A two-scale failure analysis of composite materials in presence of fiber/matrix crack initiation and propagation, Composite Structures, Volume 95, January 2013, Pages 582-597.

[38] Massart TJ, Peerlings RHJ, Geers MGD. An enhanced multi-scale approach for masonry wall computations with localization of damage. Int J Numer Methods Eng 2007;69:1022-59.

[39] Mercatoris BCN, Massart TJ. Assessment of periodic homogenisation-based multiscale computational schemes for quasi-brittle structural failure. Int J Multiscale Comput Eng 2009;7(2):153-70.

[40] Zucchini A, Lourenço PB. A micro-mechanical model for the homogenisation of masonry. Int J Solids Struct 2002;39:3233-55.

[41] Milani G, Lourenço PB, Tralli A. Homogenised limit analysis of masonry walls, Part I: failure surfaces. Comput Struct 2006;84:166-80.

[42] Milani G, Lourenço PB, Tralli A. Homogenised limit analysis of masonry walls, Part II: structural examples. Comput Struct 2006;84:181-95.

[43] Sepe V, Marfia S, Sacco E. A nonuniform TFA homogenization technique based on piecewise interpolation functions of the inelastic field. Int J Solids Struct 2013;50:725-42.

[44] Ghosh S, Lee K, Raghavan P. A multi-level computational model for multi-scale damage analysis in composite and porous materials. Int J Solid Struct 2001;38(14):2335-85.

[45] Greco F, Leonetti L, Nevone BlasiP. Adaptive multiscale modeling of fiber-reinforced composite materials subjected to transverse microcracking. Compos Struct 2014;113:249-63.

[46] Greco F, Leonetti L, Lonetti P, Nevone BlasiP. Crack propagation analysis in composite materials by using moving mesh and multiscale techniques. Comput Struct 2015;153:201-16.

[47] Lloberas-Valls O, Rixen DJ, Simone A, Sluys LJ. On micro-to-macro connections in domain decomposition multiscale methods. Comput Method Appl Mech Eng 2012;225:177-96.

[48] Greco F, Leonetti L, Luciano R. A multiscale model for the numerical simulation of the anchor bolt pull-out test in lightweight aggregate concrete. Constr Build Mater 2015;95:860-74.

[49] Feo L, Greco F, Leonetti L, Luciano R. Mixed-mode fracture in lightweight aggregate concrete by using a moving mesh approach within a multiscale framework. Compos Struct 2015;123:88-97. 
[50] Greco F., Leonetti L., Luciano R., Nevone Blasi P., An adaptive multiscale strategy for the damage analysis of masonry modeled as a composite material, Composite Structures 153 (2016) 972-988.

[51] Anthoine A. Homogenization of periodic masonry: plane stress, generalized plane strain or 3D modelling? Commun Numer Methods Eng 1997;13:319-26.

[52] Massart TJ, Peerlings RHJ, Geers MGD, Gottcheiner S. Mesoscopic modeling of failure in brick masonry accounting for three-dimensional effects. Eng Fract Mech 2005;72(8):1238-53.

[53] Lourénço P.B. Computations on historic masonry structures. Prog Struct Eng Mater 2002;4(3):301-19.

[54] L. Macorini and B. A. Izzuddin, A non-linear interface element for 3D mesoscale analysis of brickmasonry structures, Int. J. Numer. Meth. Engng 2011; 85:1584-1608.

[55] Geubelle P.H., Baylor J.S. (1998), Impact-induced delamination of composites: a 2D simulation. Composites Part B: Engineering, 29(5): 589-602.

[56] Lourenço PB, Rots JG. Multisurface interface model for analysis of masonry structures. J Eng Mech 1997;123(7):660-8.

[57] Vandoren B, De Proft K, Simone A, Sluys LJ. Mesoscopic modelling of masonry using weak and strong discontinuities. Comput Methods Appl Mech Eng 2013;255:167-82.

[58] Farhat, C., Lesoinne, M., LeTallec, P., Pierson, K., \& Rixen, D. (2001). FETI-DP: a dual-primal unified FETI method-part I: A faster alternative to the two-level FETI method. International journal for numerical methods in engineering, 50(7), 1523-1544.

[59] COMSOL AB, COMSOL Multiphysics Reference Manual, 2015.

[60] Raijmakers TMJ, Vermeltfoort AT. Deformation controlled tests in masonry shear walls - Report B-921156, technical report. Delft, The Netherlands: TNO-Bouw; 1992.

[61] Page AW, Finite element model for masonry. ASCE J. Struct. Div. 1978; 104:1267-1285.

[62] Lopez J, Oller S, Onate E, Lubliner J. A homogeneous constitutive model for masonry. Int. J. Numer. Methods Eng. 1999; 46:1651-1671. 